\title{
The Endogenous Formation of a City: Population Agglomeration and Marketplaces in a Location-Specific Production Economy*
}

\author{
Marcus Berliant ${ }^{\dagger} \quad$ Hideo Konishi ${ }^{\ddagger}$
}

January 28, 2000

* The authors wish to thank the National Science Foundation for financial support (grant SBR-9319994), the Center for Public Financial Management, Carnegie-Mellon University, for computational support and its director, Robert P. Strauss, for general discussions. We thank Clara Asnes for help with the figures. Comments and suggestions made by Masa Fujita, Tony Smith, and Jacques Thisse in the Regional Science Theory Workshop at the University of Pennsylvania were invaluable. Conversations with Jim Peck were quite helpful as well. Detailed comments from a referee and John Quigley (the editor) substantially improved the readability of the paper. We also thank Richard Arnott, John H. Boyd III, Richard Boylan, Graciela Chichilnisky, Dimitrios Diamantaras, Charles Engel, Douglas Gale, Mamoru Kaneko, Lionel McKenzie, Suzanne Scotchmer, Tomoichi Shinotsuka, Raghu Sundaram, William Thomson, Shlomo Weber, Myrna Wooders, Bill Zame, and seminar participants at the Midwest Mathematical Economics Meetings in Madison, at the Econometric Society Meetings in Quebec City, at the Southeastern Economic Theory Meeting in Charlottesville, at CORE, at the Australian National University, at the Ohio State University, at Osaka University, at Southern Methodist University, at the University of Alabama, at the University of New South Wales, at the University of Queensland, at the University of Rochester, at Washington University, and at the University of Tokyo for their comments. The authors retain full responsibility for any errors remaining in the paper.

$\dagger$ Department of Economics, Washington University, Campus Box 1208, One Brookings Drive, St. Louis, MO 63130-4899.

$\ddagger$ Department of Economics, Boston College, Chestnut Hill, MA 02467-3806. 


\begin{abstract}
Much of the literature on the endogenous generation of a city employs increasing returns to scale in order to obtain agglomeration. In contrast, the model considered here focuses on the role of marketplaces or trading centers in the agglomeration of population as cities. Gains to trade in combination with transportation and marketplace setup costs suffice to endogenously generate a city or cities with one or multiple marketplaces. It is assumed that consumers are fully mobile while production functions are location-specific. The exchange of commodities takes place in competitive markets at the marketplaces, while the number and locations of the marketplaces are determined endogenously using a core concept. Unlike the standard literature of urban economics, our model can deal with differences in geography by letting the setup costs of marketplaces and the transportation system depend on location. After showing that an equilibrium exists and that equilibrium allocations are the same as core allocations, we investigate the equilibrium number and locations of marketplaces, the population distribution, and land prices. In contrast with earlier literature, the results are general in the sense that specific functional forms are not needed to obtain existence of equilibrium, equilibria are first best, and equilibria are locally unique (in our examples).
\end{abstract}


Where human beings organize their economy around market exchange, trade between city and country will be among the most powerful forces influencing cultural geography and environmental change. The ways people value the products of the soil, and decide how much it costs to get those products to market, together shape the landscape we inhabit. (Cronon, 1991, p. 50)

\section{Introduction}

Over the past few years, increasing attention has been focused on the economics of cities. Why do cities form where they do? What are the driving forces behind the formation of cities? What roles do gains to trade and the location of marketplaces play? Is perfect competition consistent with spatial modeling?

Naturally, in order to motivate our approach to these questions, it is useful to see how the extant literature has addressed them. There are two important aspects of an explanation of why a city is formed in a particular place. These have been labeled as first and second natures of geography (Cronon (1991)). A first nature of geography is an advantage created by nature of locating at a certain place (such as a natural harbor, a river, and so on), while a second nature of geography is an advantage created by human beings of locating there.

There are many ways to interpret the notion of second nature of geography. Krugman $(1991 \mathrm{a}, \mathrm{b})$ points out that increasing returns to scale from population agglomeration are important in explaining the formation of cities, and stresses the importance of the history of a city. History affects the consumers' location choices in each period, and it determines the future of a city. Krugman also introduces transportation cost and discusses the resulting location of cities. Krugman (1993a) introduces dynamics into the model, while Krugman (1993b) uses a continuum of locations and endogenously generates potential functions. Fujita and Krugman (1995) include land explicitly in the model, allow many types of manufactured goods, and obtain various types of city configurations and industrial specializations. ${ }^{1}$ Similar models were investigated earlier by Fujita and Ogawa (1982) and Fujita (1988) as well. Jane Jacobs $(1969,1984)$ lends support to this entire line of argument.

Although it is limited to a specific functional form, the use of a Dixit-Stiglitz (1977) model in this line of research to explain city formation seems important, since it can generate various types of urbanization in developed (industrialized) countries. However, since the model features increasing returns to scale and monopolistic competition, there is a large indeterminacy of the set of equilibria. Therefore, from the same economic data (preferences, endowments, and so on), we can obtain many equilibrium city structures (different numbers of cities at different locations).

\footnotetext{
${ }^{1}$ Ciccone and Hall (1997) find a positive relationship between productivity level and the density of economic activity. Kim (1995) provides some empirical evidence on regional concentration or specialization of industrial production in the United States. For the data from the 1920's to the present, this evidence seems to contradict the verbal arguments of Krugman (1991b) concerning industrial specialization.
} 
In this paper, we will focus on the history of the formation of a city as detailed by Cronon (1991), who analyzes the birth and the development of Chicago. Before and in the early stages of industrialization, Cronon says that the second nature of geography played a crucial role in the development of Chicago. What Cronon means by "second nature" is actually something different from increasing returns to scale due to population agglomeration.

Each new improvement meant a shift in regional geography - a dredged harbor here, a canal or a road there - so the advantages sustaining the city came to have an ever larger human component. A kind of 'second nature,' designed by people and 'improved' toward human ends, gradually emerged atop the original landscape that nature - 'first nature' - had created as such an inconvenient jumble. (Cronon, 1991, p.56)

What Cronon calls the "second nature" of geography is the class of commodities called local public goods. Cronon cites a dredged harbor, a canal, a road, a railroad, and especially a marketplace as examples of the second nature of geography that contributed to the agglomeration of Chicago (Cronon, 1991, pp. 56 - 62).

To the north of Chicago is a forest that can produce lumber, while to the south is a prairie that is most suitable for producing wheat. Since Chicago established marketplaces for these commodities, many people accessed Chicago, and as a result, Chicago became the main regional market for all of these commodities. That is, the early development of Chicago was generated from gains from trade, location-specific production, and the establishment of marketplaces; see Cronon (1991, pp. 154-155). Transportation systems, canals, and railroads played important roles in the development of Chicago as well. The Illinois and Michigan Canal made Chicago accessible to the Illinois and Mississippi Rivers (Cronon, 1991, pp. 6364). A local railroad network reduced the cost of shipping lumber and crops and enlarged Chicago's regional market (Cronon, 1991, pp. 65-70). More globally, the Erie Canal and railroads linked Chicago with New York - the center of the eastern market containing a major harbor that imported European commodities. The Union Central Pacific Railroad connected Chicago with San Francisco on the west coast (Cronon, 1991, p. 70). These improvements, made by people, changed Chicago from the town with 'the muddy roads and shallow harbor' to 'the new metropolis of the Great West' (Cronon, 1991, p. 63).

This paper provides a model of city formation that focuses on marketplaces and on mass transportation systems for transporting commodities among marketplaces. We make the locations of marketplaces endogenous and we impose an individual transportation cost for accessing a marketplace as well as setup costs for both marketplaces and mass transportation systems that connect marketplaces (railroads, canals, and so on). Consumers are endowed with labor and land ${ }^{2}$ that is used either for consumption or to produce commodities. Consumers are freely mobile and they engage in production at the locations they choose. The commodities are produced using location-specific production technologies, so the amount of

\footnotetext{
${ }^{2}$ There is no absentee landlord.
} 
a commodity that is produced depends on the distribution of labor across locations. ${ }^{3}$ Consumers can trade commodities and land only at marketplaces, and they pay transportation cost individually (the opportunity cost of leisure) to access marketplaces. Hence, if there is only one marketplace, then a consumer must access it if she wants to trade goods. If there is more than one marketplace, then society must build a mass transportation system to connect these marketplaces in order to transport commodities from one marketplace to another. In this case, each consumer accesses the marketplace that is most convenient. The explicit introduction of a mass transportation system to the model makes our model rich enough to explain the development of cities in the places where terminals of a mass transportation system were located, while allowing the location of such a system to be endogenous. ${ }^{4}$ Gains to trade provide an incentive for marketplaces to coalesce. Although most of the papers in this field introduce increasing returns to scale into the production technology in order to induce agglomeration, our model focuses on cities as marketplaces or trading centers following Berliant and Wang (1993). ${ }^{5}$ That is, we introduce (local) public goods into the model instead of increasing returns to scale to obtain population agglomeration. The locations of marketplaces and consumers as well as land prices and the method of sharing the cost of setting up marketplaces and a mass transportation system are all endogenous and simultaneously determined. Since we treat marketplaces as local public goods, the collective choices made by consumers to set up marketplaces, to set up a mass transportation system, and to share the costs of these goods play an important role in the analysis. ${ }^{6}$

Moreover, since we let the setup costs of marketplaces and a mass transportation system be dependent on location, our model can capture aspects of the first nature of geography. ${ }^{7}$ For example, we can allow for the following situations: one location might be more suitable than another for dredging a harbor, or it might be less costly to build a railroad between one pair of marketplaces than another. In the previous literature, including the work with increasing returns, it is usually assumed that the geography is a homogeneous plane or line.

\footnotetext{
${ }^{3}$ Location specific production is one of the assumptions that implies gains to trade. In the context of Chicago's rise, Cronon (1991) points to location specific production of lumber, grains, and livestock in separate chapters of his book. Of course, Chicago eventually became the main regional market for all of these commodities. In terms of a theoretical predecessor, Schweizer, Varaiya and Hartwick (1976) have location specific production.

${ }^{4}$ Buffalo and Los Angeles are other examples of such cities. Buffalo became a large city because it is located at the west end of the Erie Canal. Although San Diego has a better natural harbor than Los Angeles, Los Angeles is bigger than San Diego, as Los Angeles was chosen as the terminus of a transcontinental railroad.

${ }^{5}$ Berliant and Wang (1993) construct a model of city formation without production where the location of marketplaces is endogenous and study under what conditions a monocentric city is formed. Gains to trade, transport cost, and the setup cost of marketplaces drive the model. They start from a social welfare maximization problem and find price support for the social optimum, which generates a market equilibrium. Consumers have limited mobility, as they must reside in the area where their endowments are found.

${ }^{6}$ The reasons why we must employ specialized rather than general models to study our questions are as follows. Aside from the notion that endogeneity of location as well as prices and quantities makes it difficult to establish precisely characteristics of equilibrium, the introduction of a spatial dimension causes some interesting problems in solving our model. As we shall see in Example 1, the nonemptiness of the core is not generally assured if there is heterogeneity in consumers.

${ }^{7}$ Ellickson and Zame (1994) also stress the importance of the first nature of geography.
} 
That is, the locations in the economy are physically identical ex ante. However, the role of natural harbors is certainly quite important in explaining the development of Boston and New York. Since Chicago faces on Lake Michigan, heavy lumber could easily be transported to Chicago from the north. The location-specific production technology implicitly describes part of the first nature of geography as well. Our model can capture these elements.

In the context of the models contained in the literature, there are three ways to define a city, or agglomeration. The definition can involve any combination of (i) the concentration of population (e.g. Baruchov and Hochman (1977) and Papageorgiou and Smith (1983)), (ii) the concentration of market or transaction activities (e.g. Stuart (1970), Mills (1972), Hartwick and Hartwick (1977) and Baesemann (1977)), and (iii) the concentration of employment and production (e.g. Mills (1967), Papageorgiou (1979) and Imai (1982)). Our work is concerned with all of these aspects of agglomeration. We define a city to be where population agglomerates, but both marketplace location and employment play roles in our model.

It is important to relate our work to the Spatial Impossibility Theorem of Starrett (1978), one of the central results in the field (see Fujita (1986) for an insightful discussion of this result). Loosely speaking, the Theorem states that if (i) there is no cost of relocation, (ii) consumers' preferences and firms' technologies are independent of location, (iii) the economy is closed, and (iv) each location has complete competitive markets, then there is no equilibrium with positive transportation cost in aggregate. In essence, under these assumptions, economic activity and agents are completely spread out over space if land is included in the model. Our model circumvents this result by dropping assumptions (ii) and (iv), employing location specific production and a setup cost for marketplaces.

Most models in the literature drop assumptions (ii) and/or (iv) as well: consumers must access a central business district, the location of which is exogenously given. The central business district might be the only marketplace, or the only location where goods are produced. For example, Ellickson and Zame (1991) prove very general theorems on the existence of market equilibrium and core equivalence in an exchange economy with this type of framework. In contrast, we employ location specific production and determine the locations of marketplaces endogenously. In the end, if one is to have agglomeration in equilibrium, one must dispense with one or more of the Starrett assumptions. A review of the various articles accomplishing this in different ways can be found in Berliant and Wang (1993). We note in passing that much of the literature of regional science does not violate any of the assumptions of the Starrett Theorem, and thus must rely on other forces, such as population externalities, to achieve agglomeration; see Berliant and ten Raa (1994) for further detail.

The Starrett Theorem applies when the number of consumer locations is finite, as in the model presented here. The interpretation of the conclusion of the theorem is simply that the equilibrium distribution of population (and production) is uniform across location, while each location is self-sufficient. In fact, this coincides with the conclusion of the theorem in the case where the number of locations is infinite or forms a continuum. In either class of model, population agglomeration is defined to be any distribution of population other than the uniform distribution; see Papageorgiou and Smith (1983), for instance. A uniform 
population distribution implies that consumers cohabit locations in either class of model.

Our main results are as follows. First, we consider a fixed marketplace structure and transport network (in Section 3). Under the assumption that the setup costs for marketplaces and mass transportation systems are proportional to the number of participants in a market, the core is shown to be nonempty and equivalent to the set of competitive equilibrium allocations with market utilization fees for marketplaces. At a core allocation, identical consumers are treated equally almost surely in the utility sense. The most interesting feature of the results with a fixed marketplace structure is the strength of the assumptions (particularly a boundary condition) that must be employed. The strong assumptions are needed because consumers must choose one location as residence, thus generating a nonconvexity in consumption sets. Next, in Section 4, we allow the marketplace structure and transport network to be determined endogenously. Surprisingly, if consumers are heterogeneous, the core can be empty due to a nonconvexity in the aggregate production set caused by the cost a consumer incurs in accessing a marketplace. The result is illustrated through a simple example (see figures 3 and 4 ) that is, perhaps, the most important piece of this work. ${ }^{8}$ Potential for this nonconvexity seems present in any model where it is costly for consumers to access markets. If all consumers are identical and setup costs are proportional to the number of participants in a market, the core is shown to be nonempty. Finally, if the setup costs for marketplaces and mass transportation systems are independent of the number of participants and all consumers are identical, the core is shown to be nonempty but the set of core allocations with a fixed marketplace structure is larger than the set of competitive equilibrium allocations. The characteristics of the solutions are examined through simulations. ${ }^{9}$ The numerical analysis says that as the setup cost of marketplaces goes up, the number of marketplaces decreases and population agglomeration is enhanced. The rent of land in the center of a city will increase with marketplace setup cost as well. If either preferences are asymmetric in produced goods or the production technology for marketplaces is asymmetric in produced goods, the location of marketplaces will be biased. If the setup cost of a mass transportation system is dependent on the locations of marketplaces, the equilibrium number and locations of marketplaces are affected. The last result suggests the importance of the first nature of geography in the theory of city formation.

As we shall see when examples are presented, marketplaces will sometimes be located in the same places as consumers. This will occur under symmetry when the cost of building marketplaces is low compared with the cost of accessing them. In other cases, when preferences or technology are asymmetric in goods or when the cost of building market places is high relative to access cost, marketplaces will be located apart from consumers. Due to high

\footnotetext{
${ }^{8}$ This negative example does not imply that the assumption of identical consumers is necessary to explain population agglomeration in our setting. It simply says that it is difficult to determine the locations of marketplaces endogenously, for example by using the core as a collective choice mechanism, if consumers are not identical. Once the locations of marketplaces are determined, our model explains population agglomeration even with heterogenous consumers.

${ }^{9}$ The reason we use simulations instead of analytically deriving comparative statics to characterize equilibrium is that it is very difficult to obtain analytical results since we have many endogenous location variables as well as many (utility equating) constraints due to the free mobility of consumers.
} 
land rents near marketplaces, some consumers will not locate there. Thus, benefits derived from locating near marketplaces are capitalized in land rents. Wages can also differ across locations, depending on the marginal productivity of labor.

We set up a general model and define some relevant concepts in section 2. Section 3 shows that the core given fixed locations of marketplaces is nonempty and is equivalent to a notion of equilibrium with market utilization fees. Section 4 demonstrates that the core with endogenous locations of marketplaces can be empty in general and proves the nonemptiness of the core in a special case. Section 5 characterizes core allocations using examples. Section 6 concludes. An appendix contains all of the proofs.

\section{A General Model}

Here we will construct an abstract spatial economy that contains variants of the linear city and the monocentric city models as special cases.

\subsection{An Overview of the Economy:}

There are I (finite and integer) produced consumption commodities in the economy. There is a finite number of different locations in the economy. The location set is denoted by $J \subset \Re^{m}$, where $m$ is a positive integer representing the dimension of the location space (typically, $m=1$ or 2 ) and $J$ is finite. Each location $j \in J$ is just a point, but it contains a positive amount of homogeneous land. A consumer can choose one location from $J$ to live in. Each consumer owns land and labor as her endowment. Although commodities can be transported physically among locations, land and labor are specific to locations. Each consumer produces commodities using production technologies available at her location, and brings her products to a marketplace to trade goods (commodities and land). In marketplaces, other consumers are selling goods she wants. The consumer is required to spend time to go to a marketplace; the time spent depends on the location of the marketplace and her residential location. Marketplaces can be established in a feasible marketplace location set $D \subset \Re^{m}$. The set $D$ is assumed to be compact. ${ }^{10}$ If we use a linear city model, then $D$ is an interval in $\Re$. If we use a monocentric city model, then $D$ is a subset of $\Re^{2}$. More than one marketplace can be established if needed. In such a case, marketplaces must be connected by a mass transportation system to obtain commodity flows between them. To establish a marketplace

\footnotetext{
${ }^{10}$ There is an asymmetry between the cardinality of consumer locations (finite) and potential marketplace locations (a continuum). If we modelled both as a continuum, complications would arise in both the simulations and in core equivalence, aside from much more technical proofs. If both were finite, odd locations of marketplaces would occur in the simulations (contained in section 5) simply because marketplace locations that would otherwise be optimal might be omitted from the set of potential marketplace locations. Note that we can analyze the case of finite consumer and marketplace locations by modifying our model only slightly. The reason we treat these two sets asymmetrically is that we can analyze numerically the sensitivity of the locations of markets when economic data change with the assumption that consumer locations are finite (see section 5).
} 
and a mass transportation system, a coalition of consumers has to pay costs depending on the size of the coalition using the marketplace system. Figures 1 and 2 illustrate examples of our economy when $m=1$. In the examples, $J=\{0, .33, .67,1\}$ and $D=[0,1]$. In locations $\{0, .33\}$, commodity 1 is produced, and in locations $\{.67,1\}$, commodity 2 is produced. In each $j \in J$, there is a certain (possibly different) amount of land. In Figure 1, there is only one marketplace, located at $\{.5\}$. At equilibrium, a commodity will have the same price at each marketplace. If a consumer is living at location $\{0\}$, she travels from $\{0\}$ to $\{.5\}$ in order to trade. If, instead, a consumer is living at location $\{.33\}$, she only travels from $\{.33\}$ to $\{.5\}$ in order to trade. In Figure 2, there are two marketplaces with locations $\{.167\}$ and $\{.833\}$. If a consumer is living at $\{0\}$, she accesses the marketplace $\{.167\}$, since the travel cost to that marketplace is lower. On the other hand, if a consumer is living at $\{1\}$, she accesses the marketplace at $\{.833\}$. The two marketplaces are connected by a mass transportation system that supplies commodities to each marketplace. If this transportation system did not exist, only commodity 1 would be available at the marketplace $\{.167\}$, and only commodity 2 would be available at the marketplace $\{.833\}$. A consumer chooses her location $j$ from $J$ taking into account, for each location, the wage, rent, and commuting cost to the closest marketplace.

\section{[Figures 1 and 2 around here]}

\subsection{Marketplaces:}

The location of a marketplace can be anywhere in $D$. No marketplace requires land: a marketplace is a point $d \in D$. The locations of the marketplaces are denoted by $\left\{d_{1}, d_{2}, \ldots, d_{k}\right\}=$ $\mathbf{d} \subset D$. Let $K$ be the collection of finite sets in $D(\emptyset \in K)$. If there is only one marketplace, all consumers have to access it to trade with each other. If there is more than one marketplace, then each consumer will access the marketplace that is most convenient. We assume that the transportation cost of commodities between marketplaces is negligible, while to access marketplaces consumers have to use their time endowments. This assumption appears reasonable, but we can introduce costs of transporting mass quantities of commodities between marketplaces with some change in the analysis that follows (but notation would become quite complicated; see footnote 15 and subsection 2.5). Instead, we wish to focus on the trade-off between the number of marketplaces (with a set-up cost) and the (leisure) cost of consumer access to them.

\subsection{Consumers:}

There is a continuum of consumers. ${ }^{11}$ The set of consumers is denoted $A=[0,1]$, and a representative element of $A$ is $a$. The consumers form an atomless measure space $(A, \mathcal{A}, \nu)$,

\footnotetext{
${ }^{11}$ Given the nonconvexities in the consumption sets of consumers due to the discreteness of location choice, it would be unreasonable to expect any of the results in this paper to hold without continuum of agents, except in an appropriate approximate sense when the population is large. The nonconvexity results from the restriction that consumers can enjoy private goods in one location only, so the consumption set will be
} 
where $\mathcal{A}$ is the Borel sigma algebra of $A$ and $\nu$ is Lebesgue measure on $A$. By definition, $\nu(A)=1$. Until Theorem 3, we will allow for heterogeneous types of consumers.

\subsection{Individual Transportation:}

Consumers access marketplaces to trade commodities with others. They pay a cost to bring their products to marketplaces. To keep the model simple, there are no physical or monetary transport costs; there is only the cost of time to travel to a market. We assume that travel cost (round trip travel time) from location $j$ to a marketplace $d$ is represented by $\tilde{\delta}_{j}(d)$, where $\tilde{\delta}_{j}: D \rightarrow \Re_{+}$is a continuous function. Note that this function captures Cronon's first nature of geography in accessing a marketplace, since travel cost can vary with the terrain between a consumer and a market. Travel cost is assumed to be independent of the quantity of goods transported. Since a consumer accesses a marketplace that is most convenient, time to travel to a marketplace in marketplace structure $\mathbf{d}$ for a consumer residing at $j \in J$ is given by $\delta_{j}(\mathbf{d})$, where $\delta_{j}: K \rightarrow \Re_{+}$is such that $\delta_{j}(\mathbf{d})=\min _{d \in \mathbf{d}} \tilde{\delta}_{j}(d)$. The Euclidean (round trip) distance from location $j$ to the closest marketplace given marketplace structure $\mathbf{d} \in K$ is an example of $\delta_{j}(\mathbf{d}) ;$ i.e., $\delta_{j}(\mathbf{d})=2 \min _{d \in \mathbf{d}}\|j-d\| .{ }^{12}$

\subsection{Mass Transportation among Marketplaces:}

When there is more than one marketplace, it is necessary to build a mass transportation system to have commodity flows among them. One can imagine the situation where there is a railroad station in front of each marketplace. Commodities traded in a marketplace are either brought by individual traders or transported from other marketplaces by the mass transportation system. For simplicity, we assume that there is no marginal transportation cost to bring commodities from one marketplace to another. Although the arguments below are not affected much by marginal costs in the mass transportation system, this change would introduce differences in commodity price vectors across markets. In such a case, notation would become very messy, and each consumer's choice over which marketplaces to access would depend on the price vector in each marketplace as well as the travel cost from her location to each marketplace (a consumer's choice would not be as simple as stated in the last subsection). On the other hand, we assume that the cost of building a mass transport system depends on the marketplace structure $\mathbf{d} \in K$.

a disjoint union of convex sets, one for each location. Consider the case of a fixed marketplace structure and transport network. As in Hildenbrand (1974), the core equivalence and equilibrium existence results, embodied in Theorems 3.1 and 3.2 below, will not hold without an atomless measure space of agents; it is only within this framework that Lyapunov's theorem can be applied. Of course, large finite populations can be used to obtain approximate equilibrium and approximate core equivalence. Moreover, we wanted to give the results on endogenous marketplace and transport structure, contained in Section 4, the best chance possible. This gives our example more content than one based on a finite population.

${ }^{12}$ We could replace the Euclidean norm with any other norm as well. For other types of norms, see Beckmann and Thisse (1986, pp. 59-62). 


\subsection{The Trading Set:}

It is assumed that each consumer must reside in exactly one location in $J$. Since consumers can choose their locations freely, consumption sets are nonconvex, and even disconnected. This fact does not depend on the finiteness of the location set $J$. Even if $J$ is a convex subset of $\Re^{m}$, the trading set is always nonconvex since each consumer needs to choose one location as her residence. There is a further complication. Since production is locationspecific, we cannot treat labor as a homogeneous good, since it has a different effect on output at different locations (for example, wages in different locations can be different). Thus, the usual definition of a labor endowment does not make sense. To avoid these difficulties, we treat labor in each location as a different good, and use a trading set instead of a consumption set (McKenzie (1959)). That is, we will work on net trades instead of actual consumption plans. A trading set is a consumption set where the endowment point is normalized to the origin. Let $\Omega=\Omega_{c} \times \Omega_{\ell} \times \Omega_{L} \times \Omega_{J}=\Re^{I} \times \Re^{2 J} \times J$ be the potential trading set, where $\Omega_{c}=\Re^{I}, \Omega_{\ell}=\Re^{J}$, and $\Omega_{L}=\Re^{J}$ denote potential commodity, leisure, and land trading sets, while $\Omega_{J}=J$ denotes the potential location choice set, respectively. Consumers' trading sets with no transportation costs are represented by the closed-valued measurable correspondence $X: A \rightarrow \Omega$. For simplicity, we let $X(a)=\cup_{j \in J} X_{j}(a)$, where we define $X_{j}(a)=\Re_{+}^{I} \times H_{j}(a) \times L(a) \times\{j\}$, and where $H_{j}(a)$ and $L(a)$ are the type a consumer's leisure trading set and land trading set, respectively, when she chooses location $j$. The specification of the trading set implies that no consumer is endowed with produced commodities. The $j$ th axis of $H_{j}(a) \subset \Re^{J}$ is $[-T(a), 0]$ and the other axes are all $\{0\}$, where $T(a)$ is the leisure endowment of a type $a$ consumer. By this we mean that if a consumer chooses location $j$, she can consume leisure or supply labor only at $j$. The land trading set is $L(a)=\left\{L \in \Re^{J}: L \geq-b(a)\right\}$, where $b(a) \geq 0$ denotes the initial endowment (vector) of land belonging to consumer a (in all locations). ${ }^{13}$ A typical element of $X(a)$ is denoted $(c, \ell, L, j)$, representing commodity consumption, net leisure consumption, net land consumption, and location choice, respectively. Note that $-\ell$ denotes labor supply plus transportation cost. To show core equivalence (Theorem 3.1) and the existence of equilibrium (Theorem 3.2), it is most convenient to modify the trading sets taking transportation costs (commuting costs to a marketplace) into account. Let $\tilde{X}_{j}(\mathbf{d}, a)=\Re_{+}^{I} \times \tilde{H}_{j}(\mathbf{d}, a) \times L(a) \times\{j\}$, where the $j$ th axis of $\tilde{H}_{j}(\mathbf{d}, a)$ is $\left[-T(a)+\delta_{j}(\mathbf{d}), 0\right]$; i.e., $\tilde{X}_{j}(\mathbf{d}, a)$ is obtained by truncating and translating $X_{j}(a)$ by $\delta_{j}(\mathbf{d})$. Let $\tilde{X}(\mathbf{d}, a)=\cup_{j \in J} \tilde{X}_{j}(\mathbf{d}, a)$. We call $\tilde{X}(\mathbf{d}, a)$ the translated trading set (see also Berliant and Fujita (1992)). This set is the collection of net trades accounting for the commuting cost to a marketplace.

\subsection{Preferences:}

The preference relation $R(a) \subset X(a) \times X(a)$ is assumed to be a complete preorder. $R(a)$ is closed in $X(a) \times X(a)$. To get the preference relation on the translated trading set $R(\mathbf{d}, a) \subset$

\footnotetext{
${ }^{13}$ We define vector inequalities as follows: Let $x, x^{\prime} \in \Re^{n}$. Then $x \geq x^{\prime}$ if $x_{i} \geq x_{i}^{\prime}$ for all $i=1, \cdots, n$, $x>x^{\prime}$ if $x \geq x^{\prime}$ and $x \neq x^{\prime}$, and $x>>x^{\prime}$ if $x_{i}>x_{i}^{\prime}$ for all $i=1, \cdots, n$.
} 
$\tilde{X}(\mathbf{d}, a) \times \tilde{X}(\mathbf{d}, a)$, translate the preference relation by $\delta_{j}(\mathbf{d})$. Denote $b y \succ_{a}\left(\succ_{a}^{\mathbf{d}}\right)$ and $\succeq_{a}\left(\succeq_{a}^{\mathbf{d}}\right)$ the strict and weak preference relations, respectively, induced by $R(a)$ on $X(a) \times X(a)$ $(R(\mathbf{d}, a)$ on $\tilde{X}(\mathbf{d}, a) \times \tilde{X}(\mathbf{d}, a))$. The space of admissible preferences is denoted by $\mathcal{R}$ and endowed with the topology of closed convergence (Hildenbrand (1974)). We assume that $R: A \rightarrow \mathcal{R}$ is a measurable mapping, where $\mathcal{R}$ is endowed with the Borel $\sigma$-algebra.

\subsection{Production:}

In our economy, the production structure is location-specific. Land and labor are used to produce consumption commodities. The production set that is available at location $j$ is denoted by $Y_{j} \subset \Re^{I} \times \Re^{2 J} ; Y_{j}$ is a closed convex cone with vertex at the origin. ${ }^{14}$ We assume $Y_{j} \cap \Re_{+}^{I} \times \Re_{+}^{2 J}=\{0\}$, and $\Re_{-}^{I} \times \Re_{-}^{2 J} \subset Y_{j}$. Denote the aggregate production set by $Y=\sum_{J} Y_{j}$, which is also assumed to be a closed, convex cone with vertex at the origin, and $Y \cap\left(\Re_{+}^{I} \times \Re_{+}^{2 J}\right)=\{0\}$. These assumptions imply a constant returns to scale technology. In this specification, we do not explicitly exclude the possibilities that (i) inputs (such as raw materials) are transported among locations (there is trade in inputs), and (ii) produced commodities are used as inputs for the production of other commodities (there is trade in intermediate goods). Although these possibilities do not cause any mathematical problems in our proofs, they may be inconsistent with the assumption that the marginal cost of mass transportation is zero (see subsection 2.5). ${ }^{15}$ Hence, we will exclude these cases for now; finding assumptions to exclude these possibilities is quite easy, while adjusting the model to account for these possibilities is easy but technical and cumbersome. We will return to this point in the conclusion of the paper.

\footnotetext{
${ }^{14}$ In our work, we consider location-specific constant returns to scale production. When discussing agglomeration, increasing returns to scale are often considered. In the aspatial context, core with increasing returns can be handled using a coalition production framework, such as Hildenbrand (1974, chapter 4.2). We note, however, that in such a framework the standard core equivalence and equilibrium existence results are unlikely to hold; Hildenbrand assumes that the production correspondence is convex-valued, for example. In the spatial context, one could modify the coalition production framework to include the locations of agents as an argument in the production correspondence, thus capturing location-specific production for coalitions in the definition of core. Increasing returns in each location is admissible, though to parallel our definition and the literature we have cited, a blocking coalition using a location-specific technology must start its production at zero output, and cannot take advantage of the marginal cost reduction attribute to production of the complementary coalition. The standard core equivalence and nonemptiness results are unlikely to hold in this model, for the same reasons as in the aspatial framework.

${ }^{15}$ The problem is how to introduce the transportation cost of inputs and intermediate goods. If it is costless to transport these goods from one location to another, then it is asymmetric to assume that consumers need to transport final products (commodities) to marketplaces, and that this transportation is costly. In this case, for symmetry, we need to assume that mass transportation among markets is costly (see subsection 2.5). Thus, we assume that inputs and intermediate goods are not transportable in this paper to keep the model simple.
} 


\subsection{The Setup Costs of Marketplaces and a Mass Transportation System:}

To establish a marketplace, consumers must pay the setup costs. When there is more than one marketplace, they need to pay not only the setup costs of marketplaces but also those of a mass transportation system which connects those marketplaces. A mass transportation allows commodity flows among marketplaces. ${ }^{16}$ We assume that those setup costs depend on the number (measure) of consumers who use the marketplaces and a mass transportation. Specifically, we assume that for each measurable coalition $S \in \mathcal{A}$, the commodity bundles required to establish marketplaces at $\mathbf{d} \in K$ and a mass transportation which connects them are given by the set $Q(\mathbf{d}, S)=\nu(S) \times \mathcal{Q}(\mathbf{d})$, where $K$ is the collection of finite marketplace locations, and $\mathcal{Q}: K \rightarrow \Re_{+}^{I} \times \Re_{+}^{2 J}$ satisfies (i) $\mathcal{Q}(\mathbf{d})$ is nonempty, closed and convex, (ii) $\mathcal{Q}(\mathbf{d})+\Re_{+}^{I} \times \Re_{+}^{2 J} \subset \mathcal{Q}(\mathbf{d})$ (a free disposal property) for any $\mathbf{d} \in K$. We call $\mathcal{Q}$ the input requirement correspondence for establishing a marketplace structure. ${ }^{17}$ Note that this correspondence captures Cronon's first nature of geography in setting up a marketplace: the setup cost of marketplaces can depend on the number and locations of marketplaces. As a special case, we can let $\mathcal{Q}(\mathbf{d})=\sum_{d \in \mathbf{d}} Q^{M}(d)+\sum_{d \in \mathbf{d}} Q^{S}(d)+Q^{R} \times \tau(\mathbf{d})$, where $Q^{M}(d)$, $Q^{S}(d)$, and $Q^{R}$ denote the input requirement set for setting up a marketplace at $d \in D$, setting up a station at $d \in D$, and building up one mile of railroad, respectively, and $\tau(\mathbf{d})$ is the distance among the marketplaces. An important assumption is that the setup cost of marketplaces is proportional to the size of a coalition. This assumption is used only for proving a core equivalence theorem, and is not related to the nonemptiness of the core. Note that we do not assume anything about cost allocation among consumers ex ante.

\subsection{An Economy:}

An economy $\mathcal{E}$ is a list $((A, \mathcal{A}, \nu) ; X, R ; Y, Q, J, D)$.

\subsection{Feasible Allocations and Other Definitions Concerning Allo- cations:}

A consumption allocation in $\mathcal{E}$ is a Borel integrable function $f: A \rightarrow \Omega$ such that $f(a) \in X(a)$ a.e. in $A$. The collection of consumption allocations is denoted by $\mathcal{H}$. A list $(\mathbf{d}, f)$ is called an allocation. Let $\operatorname{proj}_{(\cdot)}(*)$ denote the projection operation of $(*)$ onto $(\cdot)$. Given $(\mathbf{d}, f)$, let $\tilde{f}(\mathbf{d}, a)$ be such that if $\operatorname{proj}_{J} f(a)=j$ then $\tilde{f}_{j}^{\ell}(\mathbf{d}, a)=f_{j}^{\ell}(a)-\delta_{j}(\mathbf{d})$ and other elements of $\tilde{f}(\mathbf{d}, a)$ are the same as those of $f(a)$, where $f_{j}^{\ell}(a)$ denotes consumer $a$ 's net

\footnotetext{
${ }^{16}$ Compare with the literature on economic network systems (see Hendrick, Piccione, and Tan (1994) and Jackson and Wolinsky (1996)).

${ }^{17}$ Readers can imagine that the production of marketplaces and a mass transportation system is done at a certain location, and that the input cost includes the cost of moving materials needed for their construction to that location, as well as the cost of moving each final product to the place where it will reside.
} 
leisure consumption at $j$. This is a consumption allocation in the translated trading set. An allocation $(\mathbf{d}, f)$ in $\mathcal{E}$ is feasible if $(\mathbf{d}, f)$ satisfies:

$$
\begin{aligned}
& \tilde{f}(\mathbf{d}, a) \in \tilde{X}(\mathbf{d}, a) \text { a.e. in } A, \\
& \quad \int_{A} \operatorname{proj}_{C} \tilde{f}(\mathbf{d}, a) d \nu(a) \in Y-Q(\mathbf{d}, A),
\end{aligned}
$$

where $C=\Omega_{c} \times \Omega_{\ell} \times \Omega_{L}$, and hence $\operatorname{proj}_{C} \tilde{X}(\mathbf{d}, a)=\cup_{J}\left\{\Re_{+}^{I} \times H_{j}(\mathbf{d}, a) \times L(a)\right\}$. The collection of feasible allocations is denoted by $\mathcal{F} .{ }^{18}$

Let $\succeq_{A}$ be such that for $f, f^{\prime} \in \mathcal{H}, f \succeq_{A} f^{\prime}$ iff $f(a) \succeq_{a} f^{\prime}(a)$ a.e. in $A$. A feasible allocation $(\mathbf{d}, f) \in \mathcal{F}$ is Pareto efficient, if for any $\left(\mathbf{d}^{\prime}, f^{\prime}\right) \in \mathcal{F}$, it is not the case that $f^{\prime} \succeq_{A} f$ with $f^{\prime}(a) \succ_{a} f(a)$ for $a$ a.e. in some $S, \nu(S)>0$. The collection of Pareto efficient allocations in $\mathcal{E}$ is denoted by $P$. A consumption allocation $f \in \mathcal{H}$ is envy free in $S$ if the following condition is satisfied a.e. for $a \in S: f(a) \succeq_{a} f\left(a^{\prime}\right)$ a.e. for $a^{\prime} \in S$. The collection of (not necessarily feasible) allocations in $\mathcal{E}$ that are envy free in $A$ is denoted by $F$. An allocation $(\mathbf{d}, f)$ in $\mathcal{E}$ is individually rational if $f(a) \succeq_{a}(0, j)$ for any $j \in J$ a.e. in $A$. Note that an individually rational allocation does not need to be a feasible allocation.

\subsection{Coalitional Deviations and Core Allocations:}

Let $S \in \mathcal{A}$ be a measurable set of agents, or a coalition, which is trying to deviate from a feasible allocation $(\mathbf{d}, f)$. A feasible coalitional deviation in $\mathcal{E}$ given the locations of marketplaces $\mathbf{d}^{\prime} \in K$ is a list of $\mathbf{d}^{\prime}, S$, and an allocation $g: A \rightarrow \Omega$ that satisfies the following condition:

$$
\int_{S} \operatorname{proj}_{C} \tilde{f}(\mathbf{d}, a) d \nu(a) \in Y-Q\left(\mathbf{d}^{\prime}, S\right) .
$$

We say $\left(\mathbf{d}^{\prime}, S, g\right)$ improves upon $(\mathbf{d}, f)$ if $g(a) \succ_{a} f(a)$ a.e. in $S$.

A core allocation in $\mathcal{E}$ given the locations of marketplaces $\mathbf{d}$ is a feasible, individually rational allocation $(\mathbf{d}, f)$ such that there is no feasible coalitional deviation given locations of marketplaces $\mathbf{d}$ that improves upon $f .{ }^{19}$ The collection of core consumption allocations

\footnotetext{
${ }^{18}$ Notice that our feasibility concept is dependent on the marketplace structure, since there cannot be more than two marketplace structures simultaneously. We assume that goods cannot be transferred from one coalition to another coalition without a merger of the two coalitions into one. This is a basic assumption in cooperative game theory. The meaning of this assumption in our setting is that without sharing the same marketplace structure, consumers cannot transfer goods to others. Furthermore, notice that if $\mathbf{d}=\emptyset(\emptyset \in K)$ then there is always a feasible allocation: i.e., a constant map $f$ such that $f(a)=(0, j) \in \Omega$ for any $a \in A$, where $j \in J$.

${ }^{19}$ In defining the core in our economy, we follow the definition of the $g$-core in congestible public good economies due to Vasil'ev, Weber, and Wiesmeth (1995); for the definition of core in pure public good economies, see Foley (1970) or Roberts (1974). Since it is natural to assume that each marketplace is accessible to anyone, at a feasible allocation in the economy, each consumer should be able to access any marketplace without exclusion. That is, at a feasible allocation there cannot be two marketplace structures, each of which serves only one of two mutually exclusive groups of consumers. In other words, to correspond with the non-exclusion of consumers from marketplaces in the real world, we require that each marketplace structure be open to everybody at a core allocation. However, when we define a coalitional deviation, we suppose that the coalition can provide marketplaces which are exclusively used by the coalition participants.
} 
in $\mathcal{E}$ given locations of marketplaces $\mathbf{d} \in K$ is denoted Core $(\mathbf{d})$. A core allocation in $\mathcal{E}$ is a feasible, individually rational allocation $(\mathbf{d}, f)$ such that for any $\mathbf{d}^{\prime} \in K$ there is no feasible coalitional deviation given $\mathbf{d}^{\prime}$ that improves upon $f$. The collection of core allocations in $\mathcal{E}$ is denoted by Core.

\subsection{An Equilibrium with Market Utilization Fees:}

Due to the setup cost of marketplaces, the usual Walras equilibrium concept itself is not useful in this model. We will use a modified Walras equilibrium that includes a city manager in the equilibrium concept. An equilibrium with market utilization fees in $\mathcal{E}$ given the locations of marketplaces is a list $(\mathbf{d}, f, y, q, p)$ where $\mathbf{d} \in K, y \in Y, q \in Q(\mathbf{d}, A)$, and $p \in \triangle(\triangle$ is the unit simplex in $\Re_{+}^{I} \times \Re_{+}^{2 J}$ ) such that:

$$
\begin{aligned}
& p \cdot y \geq p \cdot y^{\prime} \quad \text { for any } y^{\prime} \in Y, \\
& p \cdot q \leq p \cdot q^{\prime} \quad \text { for any } q^{\prime} \in Q(\mathbf{d}, A), \\
& \int_{A} \operatorname{proj}_{C} \tilde{f}(\mathbf{d}, a) d \nu(a)=y-q, \\
& p \cdot\left\{\operatorname{proj}_{C} \tilde{f}(\mathbf{d}, a)\right\} \leq-p \cdot q \quad \text { a.e. in } A, \\
& \tilde{f}(\mathbf{d}, a) \succeq_{a}^{\mathbf{d}} x \quad \forall x \in \tilde{X}(\mathbf{d}, a) \text { s.t. } p \cdot\left(\operatorname{proj}_{C} x\right) \leq-p \cdot q \text { a.e. in } A .
\end{aligned}
$$

Conditions (i) and (ii) describe the profit maximizing and the cost minimizing behavior of firms and city managers given a price vector $p$, respectively. Condition (iii) is the market clearing condition. Conditions (iv) and (v) describe the consumers' utility maximizing behavior given their budget constraints. Here, the setup cost of marketplaces and a mass transportation system is financed by charging market utilization fees $p \cdot q$ paid by market participants (the RHSs of conditions (iv) and (v)). It is slightly surprising that the equilibrium market utilization fees turn out to be uniform poll taxes rather than different taxes for different types of consumers. Our notion of equilibrium and tax system are justified by a core equivalence theorem (3.1) under the assumption of a fixed marketplace structure and transport network. The collection of equilibrium allocations with market utilization fees in $\mathcal{E}$ given the locations of marketplaces $\mathbf{d} \in K$ is denoted $E(\mathbf{d})$. Note that our equilibrium notion is specific to fixed locations of marketplaces and a fixed transport network. We could employ the valuation equilibrium concept, making the locations of marketplaces endogenous (see Mas-Colell (1980), Diamantaras and Gilles (1996), and Diamantaras, Gilles, and Scotchmer (1996)). The reason that we use the notion of equilibrium given marketplace locations is to explore the relationship between the equilibrium and Core $(\mathbf{d})$, which is analyzed in the next section. We shall return to this discussion in Section 4.

Note that there is asymmetry between these two in the excludability of outsiders, just as in the literature on core in pure public good economies. 


\section{$3 \quad$ Fixed Marketplace Locations}

In this section, we will investigate the nonemptiness of Core $(\mathbf{d})$ allocations in our economy. It is convenient to establish a couple of preliminary results in order to prove the nonemptiness of and characterize the $\operatorname{Core}(\mathbf{d})$. The strategy for this section is as follows: First, we establish the equivalence between $\operatorname{Core}(\mathbf{d})$ and $E(\mathbf{d})$, and the nonemptiness of $C o r e(\mathbf{d})$ in a general model. All of the proofs are given in the appendix. First we define a couple of assumptions.

Local Nonsatiation: Given $\mathbf{d} \in K$, for any $x \in X(\mathbf{d}, a)$, for any $\epsilon>0$, there exists $x^{\prime} \in X(\mathbf{d}, a)$ such that $\left\|x-x^{\prime}\right\|<\epsilon$ and $x^{\prime} \succ_{a}^{\mathbf{d}} x$, a.e. in $A$.

This assumption is standard, and says that for any consumption plan, there is a better one nearby.

The Boundary Condition: If $(c, \ell, L, j) \in X(a)$ satisfies $c_{i}=0$ for some $i \in I$, or $\ell_{j}=-T(a)$, or $L_{j}=-b_{j}(a)$, then for any $\left(c^{\prime}, \ell^{\prime}, L^{\prime}, j^{\prime}\right) \in X(a),\left(c^{\prime}, \ell^{\prime}, L^{\prime}, j^{\prime}\right) \succeq_{a}(c, \ell, L, j)$ a.e. in $A$.

This assumption says that any interior consumption plan is at least as good as any boundary consumption plan. That is, with zero consumption of some good, a consumer can only attain their lowest indifference curve, independent of location.

The assumption is particularly important in obtaining the closed graph property of the demand/location choice correspondence of each consumer. The closed graph property is a crucial ingredient for Kakutani's fixed point theorem when we prove existence of an equilibrium (Theorem 3.2). Since consumers must choose locations as well as bundles, their budget correspondence may not be (lower hemi-) continuous due to the nonconvexity of consumption sets. As a result, the Berge maximum theorem cannot be applied to obtain the closed graph property of demand correspondences. The boundary condition is one of the simplest assumptions that allows us to avoid this difficulty (see Mas-Colell (1977)).$^{20}$

For all $\mathbf{d} \in K$, Core $(\mathbf{d})=E(\mathbf{d})$ under local nonsatiation and the boundary condition.

The proof of the theorem is a modification of Hildenbrand's (1974). However, the boundary condition is an unusual assumption in the context of core equivalence theorems. This assumption is used in two ways. First, it gives incentives to consumers to participate in markets. Second, it is used to prove that a minimum expenditure equilibrium is an equilibrium with participation fees. Due to the lack of convexity of trading sets, a minimum expenditure equilibrium is not even a quasi-equilibrium. Hence, neither irreducibility nor monotonicity of preferences helps in proving core equivalence (and nonemptiness of $E(\mathbf{d})$ ), since there are

\footnotetext{
${ }^{20}$ Diamantaras, Gilles and Scotchmer (1996) decentralize a Pareto efficient allocation in a discrete public projects economy without using the boundary condition (the assumption that private goods are essential). Although our economy is substantially more complicated than theirs (consumers can choose their locations freely in our model), it may be possible to weaken the boundary condition if the only objective is to prove core equivalence, Theorem 3.1, and not existence of equilibrium, Theorem 3.2 (see Remark 1).
} 
multiple wealth levels that have consumption plans without cheaper points. Assumption C.3 together with C.2 in Wooders (1980) in the context of a local public good economy is very close to our boundary condition.

From Theorem 3.1, we can analyze a core allocation using the notion of equilibrium with participation fees. This argument provides the following useful observation:

Let $S \in \mathcal{A}$ be a set of consumers in which each consumer has the same preference relation and trading set with $\nu(S)>0$. Under the assumptions of Theorem 3.1, at any core allocation consumers are envy free a.e. in $S$.

Corollary 1 is important, since it establishes equal treatment (in utility) of identical consumers (in the almost everywhere sense) at core allocations. Next, we prove the nonemptiness of $E(\mathbf{d})$ and Core $(\mathbf{d})$. We need a couple more assumptions.

Interiority: There exists $j \in J$ such that:

$$
\operatorname{int}(Y-Q(\mathbf{d}, A)) \cap \int_{A} \operatorname{proj}_{C} \tilde{X}_{j}(\mathbf{d}, a) d \nu(a) \neq \emptyset .
$$

This assumption implies that the set of feasible allocations with non-zero consumption is nonempty for some distribution of consumers across locations. It assures existence of a cheaper point.

Monotonicity in Commodity Consumption: For any $j \in J$, for any $(c, \ell, L, j) \in X(a)$ such that $c \gg 0, \ell_{j}>-T(a)$ and $L_{j}>-b(a)$, if $c^{\prime}>c$ then $\left(c^{\prime}, \ell, L, j\right) \succ_{a}(c, \ell, L, j)$ a.e. in $A$.

Monotonicity in commodity consumption implies local nonsatiation in the interior of trading sets for any $\mathbf{d} \in K$.

For all $\mathbf{d} \in K$ such that $(Y-Q(\mathbf{d}, A)) \cap \operatorname{proj}_{C} \tilde{X}_{j}(\mathbf{d}, a) \neq \emptyset$ a.e. in $A, E(\mathbf{d}) \neq \emptyset$ under the boundary condition, monotonicity in commodity consumption, and interiority.

Each of these three assumptions plays an important role in the proof. Since consumption sets are disconnected (see subsection 2.6), we do not have continuity of budget relations. Interiority and monotonicity in commodity consumption assure positive commodity prices, which implies that (location-specific) price vectors are nonzero at any location. All three properties are used to prove that individual demand correspondences have closed graphs. Completeness and transitivity of $\succeq_{a}$ are also important in this part of the proof (see Konishi (1996)). We cannot use a dispersed wealth distribution type of assumption (see Mas-Colell (1977) or Yamazaki (1978) for a definition), due to Example 1 below. Note that in this theorem we allow for the possibility of empty locations. Monotonicity in commodity consumption assures that there exists a commodity with a positive price at every location.

For all $\mathbf{d} \in K$ such that $(Y-Q(\mathbf{d}, A)) \cap \operatorname{proj}_{C} \tilde{X}_{j}(\mathbf{d}, a) \neq \emptyset$ a.e. in $A, \operatorname{Core}(\mathbf{d}) \neq \emptyset$ under the boundary condition and monotonicity in commodity consumption.

Interiority is not needed to prove Core $(\mathbf{d}) \neq \emptyset$. 


\section{Endogenous Marketplace Locations}

So far, we have fixed locations of marketplaces, and analyzed the relationship between Core $(\mathbf{d})$ and $E(\mathbf{d})$. In this section, we make the marketplace structure endogenous by allowing consumers to determine the number and locations of marketplaces. The collective choice mechanism (or solution concept) we use is Core. To do that, we need to introduce a few assumptions about the setup costs of marketplaces and the mass transportation system.

We first induce a topology on $K$ that is used to define continuity properties of functions and correspondences with domain $K$. It is defined as follows. We can partition $K=\cup_{\ell=0}^{\infty} K_{\ell}$, where $K_{\ell}$ is the set of elements of $D$ with cardinality $\ell$. We can induce a topology on $K_{\ell}$ using a metric defined in the following manner. Let $\mathcal{S}(\mathbf{d})$ be the set of permutations of $\mathbf{d}$, and let $s(\mathbf{d})$ be a representative element of $\mathcal{S}(\mathbf{d})$. For any $\mathbf{d}, \mathbf{d}^{\prime} \in K_{\ell}$, the metric distance between $\mathbf{d}$ and $\mathbf{d}^{\prime}$ is defined by $\min _{s(\mathbf{d}) \in \mathcal{S}(\mathbf{d})} \sum_{k=1}^{\ell}\left\|s_{k}(\mathbf{d})-\mathbf{d}_{k}^{\prime}\right\|$, where $s_{k}(\mathbf{d})$ and $\mathbf{d}_{k}^{\prime}$ denote the $k$-th elements of $s(\mathbf{d})$ and $\mathbf{d}^{\prime}$, respectively. Taking the disjoint union of the spaces $K_{\ell}$ $(\ell=0,1,2, \ldots)$, we obtain a topological space $(K, \mathcal{O})$, where $\mathcal{O}$ is the topology on $K$ thus defined. Note that the function $\delta^{j}$ is continuous on the topological space $(K, \mathcal{O})$. From now on, the topology induced on $K$ is always taken to be $\mathcal{O}$. We assume that $\mathcal{Q}: K \rightarrow \Re_{+}^{I} \times \Re_{+}^{2 J}$ is continuous. ${ }^{21}$ As we assumed in $2.9, Q(\mathbf{d}, S)=\nu(S) \times \mathcal{Q}(\mathbf{d})$ is a nonempty, closed and convex set.

Unfortunately, there is an unpleasant example related to the nonemptiness of Core even under continuity of $\mathcal{Q}$. If consumers are not homogeneous then Core can be empty, although it is not difficult to show that $\operatorname{Core}(\mathbf{d})$ is nonempty.

${ }^{22}$ Let $D=[0,1]$. Let location 1 be at $\{0\}$, and let location 2 be at $\{1\}$. There are two types of consumers (1 and 2); the measure of each type is $\frac{1}{2}$. There are two commodities in the economy, and commodity $i(i=1,2)$ is produced in location $i(i=1,2)$. There is no land in the economy, and each type of consumer owns 2 units of leisure as endowment regardless of location choice. To set up a market, a coalition $S$ must pay $\frac{v(S)}{2}$ units of location-specific labor in both locations. The cost of setting up two marketplaces is twice as much as the cost of setting up one marketplace (there is no setup cost for a mass transportation system). There is no first nature of geography in the example. The individual (marginal) transportation cost is one. Each commodity is produced using a location-specific production set $y_{i}+\ell_{i} \leq 0$ for $i=1,2$, where $y_{i}$ denotes the output of commodity $i$. Consumers have Leontief-type utility functions: type 1 and type 2 consumers' utility functions are $u^{1}\left(c_{1}, c_{2}\right)=\min \left\{2 c_{1}\right.$, $\left.c_{2}\right\}$, and $u^{2}\left(c_{1}, c_{2}\right)=\min \left\{c_{1}, 2 c_{2}\right\}$, respectively. That is, neither type of consumer obtains utility from leisure consumption. In this economy, Core is empty.

\footnotetext{
${ }^{21}$ In the example described in subsection 2.9 , we can make the correspondence $\mathcal{Q}(\mathbf{d})$ continuous by defining $\tau(\mathbf{d})$ to be a continuous function on $(K, \mathcal{O})$ : Continuity of $\tau$ is guaranteed if (i) $\tau(\mathbf{d})=\min _{d^{\prime} \in D} \sum_{d \in \mathbf{d}}\left\|d-d^{\prime}\right\|$,

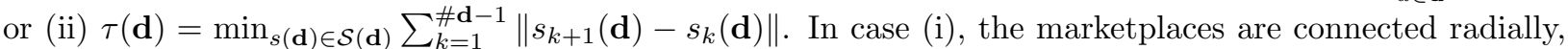
while in case (ii) the marketplaces are connected by a piecewise linear curve.

${ }^{22}$ This example is not a counterexample in the strict sense, as it violates the boundary condition. We employ this example for expositional purposes. Using the same method as in Example 1, we can construct a formal counterexample (satisfying the boundary condition) by employing the two types of preferences used in the numerical examples in the next section.
} 


\section{[Figures 3 and 4 around here]}

To see this, first notice that there can be only one marketplace in the economy. If there is no marketplace in the economy, then no one can get positive utility. If there are two marketplaces, then even if there is no individual transportation cost, again no one can get positive utility due to the setup costs of marketplaces and of the mass transportation system. Therefore, if there exists a core allocation, it must have only one marketplace. Denote the location of the marketplace by $d \in D$, which denotes the distance from location 1 . Denote the population at location 1 by $m \in[0,1]$. Then, the output vector can be written as $\left(y_{1}, y_{2}\right)=((2-d) m,(1+d)(1-m))$, for the following reason. Each consumer at location 1 can spend $2-d$ units of labor in production of commodity 1 , since she has 2 units of labor as her endowment and she needs to spend $d$ units of it for personal transportation. Since measure $m$ consumers are living at location 1, the production of commodity 1 is written as $(2-d) m$. The production of commodity 2 can be calculated in the same way: $(2-(1-d)=$ $1+d)$. Now, the cost of setting up a marketplace at $d$ is $\left(\frac{1}{2}\right.$ unit of each of commodities 1 and 2). We must subtract the cost from the production vector in order to find how much consumers can actually consume. Thus, the net production vector (or the consumption vector) given $d$ and $m$ is as follows. If $d=\frac{1}{2}$, then $\left(x_{1}, x_{2}\right)=\left(\frac{3}{2} m-\frac{1}{2}, \frac{3}{2}(1-m)-\frac{1}{2}\right)$, and the production possibility frontier (given $d=\frac{1}{2}$ ) is written as $x_{1}+x_{2}=\frac{1}{2}$. The (net) production allocation is determined by the population distribution over locations, $m$. If $d$ takes a different value, then the production possibility frontier changes (see Figure 3 ). As a result, taking an envelope of production possibility frontiers over $d$, we obtain the curve AB. Each point on $\mathrm{AB}$ corresponds an element $d \in D$. By calculation, we can show that type 1 and 2 consumers' ideal locations for the marketplace are $d^{1}=-4+\frac{1}{2} \sqrt{78}$ and $d^{2}=5-\frac{1}{2} \sqrt{78}$, respectively. That is, by allocating the population properly, type $i$ consumers can attain their most preferred consumption vector $C^{i}$ by forming a homogeneous coalition consisting of consumers of only their type distributed over both production locations (see Figure 4). Since $d^{1}$ and $d^{2}$ are different, for any $d \in D$, at least one type of consumer will deviate to improve upon the original allocation. Therefore, the core is empty. In fact, any convex combination of $C^{1}$ and $C^{2}$ is infeasible; see, for example, the point $E$ in Figure 4 . How about the case where each group forms its own marketplace and no consumer accesses the marketplace established by the other group? Unfortunately, even in this case, some type 1 consumers living in location 2 want to access the marketplace $d^{2}$, since it is closer to location 2. Then both types of consumers use both marketplaces, and they must pay doubled setup costs. Since we assume that each marketplace is accessible by anybody without exclusion, we cannot obtain a stable allocation (see the discussion of the definition of core in footnote 19).

The problem that this example suggests is that if there are transportation costs, the production possibility sets with endogenous location of marketplaces are nonconvex in general. This is the source of the trouble. Although we assume Leontief preferences in this example to make the point clear, it is not essential to the robustness of the example.

Due to this unpleasant counterexample, our theorem on the nonemptiness of the core requires identical consumers. In a strange way, this aids in justifying the use of a continuum 
of consumers in this model. Berliant (1985) points out that, under some conditions, the use of both land and a continuum of consumers in a model creates problems with consistency of the model. When all consumers are identical, however, the arguments of Papageorgiou and Pines (1990) apply. Although these arguments are not entirely convincing (see Berliant and ten Raa (1991)), they do provide some justification for our framework. One more assumption is needed before we can state our main result.

Boundedness of Feasible Marketplace Structures: There exists $\ell$, finite and integer, such that for all $\ell^{\prime} \geq \ell$, for all $\mathbf{d} \in K_{\ell^{\prime}},(Y-Q(\mathbf{d}, A)) \cap \int_{A} \tilde{X}(\mathbf{d}, A) d \nu(a)=\emptyset$.

This assumption says that all the resources in the economy can only generate a finite number of marketplaces. Since there is only a finite number $(\# J)$ of locations for consumers, the optimal number of marketplaces is always less than or equal to $\# J$ if the total marketplace setup cost increases by adding one more marketplace. In such a case this assumption is satisfied trivially.

Suppose that consumers are identical a.e. in $A$. Then, Core $\neq \emptyset$ under the boundary condition, monotonicity in commodity consumption, and boundedness of feasible marketplace structures.

The following is a characterization of Core, which is proved by using an argument in the proof of Theorem 3:

If all consumers are identical a.e. in $A$, Core $=F \cap P$.

This corollary says that the allocations in the core (up to a.e. equivalence) are the equal treatment Pareto efficient allocations. This characterization of Core simplifies the intuition behind our solution concept. Given a marketplace structure, a transportation network, and local production, Theorems 3.1 and 3.2 and their corollaries show what a market equilibrium looks like when the marketplace structure is exogenously given. Theorem 4.1 shows how a marketplace structure and transportation network are chosen so that no coalition of consumers can improve its situation by building a separate structure and network, given that population is mobile. (This is not to say that, given consumer locations, marketplaces are chosen.) That is, no coalition can improve on a Core allocation by establishing its own trading system. Corollary 3 is useful in that the search for Core allocations is the same as the search for envy-free and efficient allocations. The latter allocations are easier to find, since they involve equality constraints for utility levels across locations rather than inequality constraints for utility from coalitional deviations. The examples of section 5 exploit this structure for computational purposes, and illustrate the consequences of the Core solution concept. In essence, the solution concept implies efficiency of the allocations and equal treatment (in utility) of consumers across and within locations when setting up a trading system.

In our model, marketplace and mass transportation setup costs have been assumed to be proportional to the size of the coalition using it. This is an extreme case. The other extreme case is that irrelevant of the size of coalitions, the marketplace and mass transportation setup costs are fixed. In such a case, it is harder for coalitions to deviate from a proposed 
allocation. Thus, the core in the proportional setup cost case is a subset of the core in the fixed setup cost case. Hence, nonemptiness of the core in the fixed setup cost case is easy to show.

Suppose that consumers are identical a.e. in $A$. If marketplace setup cost is not a function of the population using the marketplaces and mass transportation system, then Core $\neq \emptyset$, but the equal treatment property does not hold.

The reason why the equal treatment property does not hold is because $\operatorname{Core}(\mathbf{d}) \neq E(\mathbf{d})$ when the marketplace setup cost is constant, independent of its user population. In such a case, the power of deviating coalitions is reduced because there is a higher setup cost. As a result, the core becomes larger than in the proportional setup cost case.

We do not proceed to analyze equilibrium and core equivalence in the model with endogenous determination of marketplace structures and transportation networks. There are two reasons. First, it is clear that the Core can be empty in many circumstances, and results characterizing the empty set are rather uninteresting. Second, the natural equilibrium concept in this context is that of valuation equilibrium. Example 2.3 of Diamantaras, Gilles, and Scotchmer (1996) shows that a Pareto optimum (for example, an element of Core) might not be decentralizable as a valuation equilibrium unless the prices of private goods depend on d. In our less abstract setting, such a price system would be too far removed from realism, in our view.

At this point, it is convenient to remark upon the differences between our work and the literature on public projects and valuation equilibrium that we have already cited. There are two that are evident. First, and of lesser significance, is the normative solution concept employed. We have used the core here, while the literature on pure public projects tend to use Pareto optimum. Second, and of more importance, is the presence of location in the model. In the public project literature, it is always assumed that consumption sets are convex once a public project is chosen. In our model, this is not the case. Even after a marketplace structure is chosen, consumers are still able to choose their locations. Thus, consumption sets are nonconvex. This framework is what leads us to use a strong boundary condition as well as a continuum of consumers. Try as we might, we have been unsuccessful at weakening the boundary condition; for further discussion in a related model, see Konishi (1996).

\section{City Formation: Examples}

In this section, we will characterize core allocations using numerical methods. We do not use comparative statics for this purpose for the reason given in footnote 9. The computations use a nonlinear programming package, GAMS/MINOS version 2.25. The basic model is the one that is described in subsection 2.1 and in Figures 1 and 2. Locations are one dimensional and contained in the unit interval $[0,1]$. There are four locations for consumers, $J=\{0,0.33,0.67,1\}$. For convenience, we call these locations $a, b, c$, and $d$, respectively. Commodity 1 is produced in $a$ and $b$ while commodity 2 is produced in $c$ and $d$. The feasible location set for marketplaces is $D=[0,1]$. The leisure endowment $T$ is set at 2 , and 
the land endowment is 1 at each location (for each consumer). Individual travel cost to a marketplace is the opportunity cost of leisure, and is the distance from a consumer's residence to the marketplace. The preferences of consumers are represented by a Cobb-Douglas type utility function, $U(c, \ell, L, j)=\left(c_{1}\right)^{\alpha_{1}}\left(c_{2}\right)^{\alpha_{2}}\left(2+\ell_{j}\right)^{\alpha_{3}}\left(1+L_{j}\right)^{\alpha_{4}}$, where $\alpha_{1}+\alpha_{2}+\alpha_{3}+\alpha_{4}=1$. The production function at $j$ is simple, and is denoted by $y_{j}=\left(-\ell_{j}\right) \times m_{j}$, where $y_{j}$ and $m_{j}$ denote the production level at $j$ and the population (measure) at $j$, respectively. The setup cost of a marketplace is represented by a kind of composite good of commodities 1 and 2. To produce one marketplace, we need $\bar{q}$ units of the composite commodity. That is, the level of $\bar{q}$ represents the setup cost of a marketplace. The production function that produces the composite good is denoted $\bar{q}=\left(c_{1}\right)^{\gamma}\left(c_{2}\right)^{1-\gamma}$. That is, we only use the two commodities that are outputs to produce the composite good. In the first three examples, we do not introduce a setup cost for a mass transportation system, and we assume that if there are $k$ marketplaces then the cost of producing marketplaces is $k$ times the cost of producing one marketplace. We will use the following sets of parameters. (Case-I): $\alpha_{i}=0.25$ for $i=1,2,3,4$, and $\gamma=0.5$. (Case-II): $\alpha_{1}=0.2, \alpha_{2}=0.3, \alpha_{3}=\alpha_{4}=0.25$, and $\gamma=0.5$. We tried many values for $\bar{q}(\bar{q}=0.01,0.05,0.1,0.2,0.3,0.4,0.5)$; it is a very important parameter in our model, since marginal travel cost is normalized to one.

(Case-I): The results are summarized in the following table (Table 1): ${ }^{23}$

\begin{tabular}{|c|c|c|c|c|c|c|}
\hline $\bar{q}$ & $\#$ & location & population & production & rent \\
\hline .01 & 4 & $a, b, c, d$ & .250 .250 .250 .250 & .34 .34 .34 .34 & .024 .024 .024 .024 \\
\hline .05 & 2 & $b, c$ & .194 .306 .306 .194 & .23 .43 .43 .23 & .015 .027 .027 .015 \\
\hline .1 & 1 & .5 & .188 .312 .312 .188 & .20 .40 .40 .20 & .013 .026 .026 .013 \\
\hline .2 & 1 & .5 & .179 .321 .321 .179 & .20 .44 .44 .20 & .011 .023 .023 .011 \\
\hline .3 & 1 & .5 & .167 .333 .333 .167 & .20 .48 .48 .20 & .008 .021 .021 .008 \\
\hline .4 & 1 & .5 & .150 .350 .350 .150 & .19 .52 .52 .19 & .006 .019 .019 .006 \\
\hline .5 & 1 & .5 & .125 .375 .375 .125 & .17 .59 .59 .17 & .004 .016 .016 .004 \\
\hline
\end{tabular}

Table 1

Commodities 1 and 2 are treated symmetrically in this case. One immediate observation is that when $\bar{q}$ is small ( $\bar{q}=0.01$ or 0.05$)$ there are many marketplaces ( 4 and 2 , respectively), but if $\bar{q}$ becomes larger $(\bar{q} \geq 0.1)$, there is only one marketplace. The population in the middle $(b, c)$ increases as $\bar{q}$ increases. The rents in the center area relative to those in the outer area also increase with $\bar{q}$. The reason why the absolute values of rents go down as $\bar{q}$ increases is that the production technology to produce marketplaces uses commodities but not land as inputs. As $\bar{q}$ goes up, more of the commodity production is used for marketplace production. Hence, commodities become scarce and the relative prices of land go down as $\bar{q}$ increases. One interesting feature is that when there are two marketplaces $(\bar{q}=0.05)$ we

\footnotetext{
${ }^{23}$ The locations of marketplaces when $\bar{q}=0.05$ can be any of $\{a, c\},\{a, d\},\{b, c\}$, or $\{b, d\}$. We choose $\{b, c\}$ only because of the nice contrast with other parameter values. The same applies to Table 2.
} 
still have agglomeration of consumers, although we also have a symmetric situation within each area producing a commodity, $\{a, b\}$ and $\{c, d\} .{ }^{24}$

(Case-II): The results are summarized in the following table (Table 2).

\begin{tabular}{|c|c|c|c|c|c|c|}
\hline $\bar{q}$ & $\#$ & location & population & production & rent \\
\hline .01 & 4 & $a, b, c, d$ & .224 .224 .276 .276 & .30 .30 .38 .38 & .020 .020 .028 .028 \\
\hline .05 & 2 & $b, c$ & .180 .268 .333 .220 & .20 .36 .45 .25 & .014 .025 .035 .020 \\
\hline .1 & 1 & .67 & .156 .273 .350 .221 & .15 .32 .50 .26 & .010 .022 .029 .016 \\
\hline .2 & 1 & .67 & .150 .287 .355 .209 & .15 .36 .53 .26 & .009 .022 .026 .013 \\
\hline .3 & 1 & .67 & .140 .304 .362 .193 & .15 .40 .57 .25 & .007 .019 .022 .010 \\
\hline .4 & 1 & .66 & .128 .327 .374 .172 & .14 .48 .61 .24 & .005 .017 .019 .007 \\
\hline .5 & 1 & .62 & .109 .357 .393 .142 & .13 .52 .67 .20 & .003 .015 .017 .004 \\
\hline
\end{tabular}

Table 2

The main difference from Case-I is the location of marketplaces when the number of marketplaces is one. Since consumers prefer commodity 2 to commodity 1 in Case-II, more of commodity 2 should be produced. This requires that a larger portion of consumers live in $c$ and $d$. Then, to save individual transportation cost, the location of marketplaces is biased to the right. Therefore, in the cases $\bar{q}=0.1,0.2,0.3$, the location of the marketplace is $c(0.67)$. Consider next the cases when $\bar{q}=0.4$ or 0.5 . The location of the marketplace is pulled toward $b$ a little because of a 'symmetric' marketplace production function $(\gamma=0.5)$. As $\bar{q}$ becomes larger, the economy is required to produce more of both types of commodity in order to produce marketplaces. In such a case, a larger and larger portion of consumers is required to live in $a$ or $b$. Then, if the location of the marketplace is very biased to the right, the total transport bill is high. Thus, it moves left as $\bar{q}$ increases. This is the source of the pulling power of the location of the marketplace.

We give a couple more examples. The next case (Case-III) is one where consumers' preferences are symmetric $\left(\alpha_{1}=\alpha_{2}=\alpha_{3}=\alpha_{4}=0.25\right)$, while the technology for production of a marketplace is asymmetric $(\gamma=0.3)$. We only provide the core allocation for $\bar{q}=0.1$. The location of the only marketplace is at 0.605 , and the distributions of population, output, and rent are as follows: $0.174,0.295,0.327,0.205$ (population); 0.168, 0.359, 0.446, 0.228 (output); $0.012,0.025,0.027,0.014$ (rent). Comparing this result with the case $(\bar{q}=0.1)$ in Table 1, we can say that if the technology for marketplace production is asymmetric, the location of the marketplace is biased toward the region that produces the commodity that is needed more in the production of marketplaces.

\footnotetext{
${ }^{24}$ This is because in this example there are only two locations producing each commodity. If we add more locations between a and $b$ and between $c$ and $d$, the locations of marketplaces converge to symmetric positions when there is no mass transportation system setup cost.
} 
Finally, we focus on the setup cost for a mass transportation system. In the next two examples, we assume that the setup cost for marketplaces $(\bar{q})$ is constant independent of the number of marketplaces, while the setup cost of a mass transportation system depends on the locations of marketplaces and the distances among them. To produce a mass transportation system, the same composite commodity as in marketplace production is used. To focus on the first nature of geography in setting up a mass transportation system, we assume $\alpha_{i}=0.25$ for all $i$, and $\gamma=0.5$. To provide marketplaces, consumers must pay $\bar{q}$ independent of the number of marketplaces. In (Case-IV), the setup cost of a mass transportation system in terms of the composite good is $3 \times \bar{q}$ per unit distance. In (Case-V), the setup cost for a mass transportation system in terms of the composite good is $9 \times \bar{q}$ per unit distance if it is built in the interval $[0,0.5]$, and is $0.3 \times \bar{q}$ per unit distance otherwise. The reader can imagine a situation in which the interval $[0,0.5]$ is mountainous, where it is costly to build a railroad, while the interval $(0.5,1]$ is a prairie, where it is less costly to build a railroad. For these two cases, we investigated only the value $\bar{q}=0.05$. In (Case-IV), we get two marketplaces $\{b, c\}$, while population, output, and rent distributions are exactly the same as those in the case $\bar{q}=0.05$ in Table 1 . For (Case-V), we obtain three marketplaces $\{0.5, c, d\}$, while population, output, and rent distributions are: $0.183,0.295,0.261,0.261$ (population); $0.187,0.375,0.357,0.357$ (output); 0.015, 0.028, 0.022, 0.022 (rent). Aside from the setup cost of a mass transportation system, (Case-IV) and (Case-V) are the same, so we can see that the first nature of geography helps determine the number and locations of marketplaces and population agglomeration, as well as the rent distribution.

\section{Conclusion}

We have seen in this paper how gains to trade, location-specific production and the setup costs of marketplaces and a mass transportation system can be used to generate agglomeration and city formation. ${ }^{25}$ Commodities are produced only by labor and the land available at each location. Commodities are transported from residences to marketplaces by individual consumers. However, this specification is unrealistic in industrialized economies. Raw materials as well as intermediate goods are traded over locations. Fortunately, our model is rich enough to capture these features, but only with further complications. We can introduce transportation technologies for moving goods between production or residential locations and a marketplace, in addition to mass transportation technologies for moving goods among marketplaces, following Schweizer, Varaiya, and Hartwick (1976). These transportation technologies are described as production set correspondences from the set of marketplace structures $(K)$ to the commodity space. By adding transportation technologies to location-specific production technologies, we can obtain net location-specific production set correspondences from the set of marketplace structures to the commodity space. Using this method, we can introduce input and intermediate good trade into our model consistently without changing

\footnotetext{
${ }^{25}$ Although our model is based on gains to trade derived from the exchange of commodities, we could alternatively make the simple reduced form assumption that consumers must access marketplaces.
} 
the basic arguments.

The model we have used can be considered complementary to the models of city formation based on increasing returns to scale from population agglomeration. In a model with increasing returns to scale, the history of a city is important in the development of a city. An important question that should be asked is: what determines the initial population distribution in the economy? The answer to this question gives us not only the initial state of the economy, but also determines the resulting development of cities over time through the mechanisms described by models employing increasing returns. Our model might reduce the "indeterminacy" problem of the increasing returns model by determining the initial population distribution and city structure. In essence, our model is driven by the same forces that drive classical international trade theory, while the models used in the spatial increasing returns literature are driven by the same forces that drive the new international trade theory.

Although we can say that our model, in combination with increasing returns models, has the potential to explain the history of a city and the resulting city structure, we have not investigated the dynamics of the development of cities explicitly. Completion of a theory of city formation seems to be an important project. ${ }^{26}$ At the very least, it can be said that unlike the previous literature, our model can account for Cronon's first nature of geography in explaining where cities locate. An interesting variation of our model would make population endogenous by adding immigration. Although the setup costs for marketplaces and a mass transportation system are rather abstract in our model, we might be able to obtain more results by specifying these costs explicitly. These ideas seem worthy of further investigation.

\section{Appendix}

\subsection{Proof of Theorem 3.1.}

First note that without setting up marketplaces, by the boundary condition, consumers will obtain the worst possible outcome. Hence, Core $(\mathbf{d})$ and $E(\mathbf{d})$ are always individually rational. Second, we prove $E(\mathbf{d}) \subset$ Core $(\mathbf{d})$. Suppose $(\mathbf{d}, f) \in E(\mathbf{d})$ but $(\mathbf{d}, f) \notin$ Core $(\mathbf{d})$. Then there exists a coalition $S \in \mathcal{A}, \nu(S)>0$, and there exists an allocation $(\mathbf{d}, g)$ such that (i) $\tilde{g}(\mathbf{d}, a) \succ_{a}^{\mathbf{d}} \tilde{f}(\mathbf{d}, a)$ a.e. in $S$, and (ii) $\int_{S} \operatorname{proj}_{C} \tilde{g}(\mathbf{d}, a) d \nu(a) \in Y-Q(\mathbf{d}, S)$. By (i), $p \cdot\left(\operatorname{proj}_{C} \tilde{g}(\mathbf{d}, a)\right)>p \cdot(y-q)=-p \cdot q$ a.e. in $S$. This implies $p \cdot\left(\int_{S} \operatorname{proj}_{C} \tilde{g}(\mathbf{d}, a) d \nu(a)\right)>$ $\max _{y \in Y} p \cdot(Y-Q(\mathbf{d}, S))=\nu(S) \times\left\{\max _{y \in Y} p \cdot(Y-Q(\mathbf{d}, A))\right\}$, which contradicts (ii). Hence $E(\mathbf{d}) \subset \operatorname{Core}(\mathbf{d})$.

Next, we will prove the most difficult part of the theorem, Core $(\mathbf{d}) \subset E(\mathbf{d})$. First, we will demonstrate the existence of a unique individual profit function. Let $\triangle^{*}=\triangle \cap Y^{*}$, where $Y^{*}$ is denotes the dual cone of $Y$. Given $p \in \triangle^{*}$, let $\Pi(\mathbf{d}, S, p)=\max p \cdot y$ s.t. $y \in Y-Q(\mathbf{d}, S)$, and let $\pi(\mathbf{d}, p)=\max p \cdot y$ subject to $y \in Y-Q(\mathbf{d}, A) \cdot{ }^{27}$ Note that $\Pi(\mathbf{d}, S, p)=\int_{S} \pi(\mathbf{d}$,

\footnotetext{
${ }^{26}$ Palivos and Wang (1996) provide a dynamic model of city formation based on an endogenous growth framework.

${ }^{27}$ In the appendix, $y$ sometimes represents an element of $Y-Q(\mathbf{d}, A)$ (the net production set) instead of $Y$ (the private production set). This is just for notational simplicity, and the reader should be able to
} 
$p) d \nu$, since $Q(\mathbf{d}, S)=\nu(S) \times Q(\mathbf{d}, A)$. Therefore, $\pi(\mathbf{d}, p)$ is a Radon-Nikodym derivative of $\Pi(\mathbf{d}, S, p)$, which is unique up to $\nu$-equivalence.

Let $(\mathbf{d}, f) \in \operatorname{Core}(\mathbf{d})$. Define a correspondence $\psi$ from $K \times(A, \mathcal{A}, \nu)$ into $\Omega$ by $\psi(\mathbf{d}, a)=$ $\left\{x \in \tilde{X}(\mathbf{d}, a): x \succ_{a}^{\mathbf{d}} \tilde{f}(\mathbf{d}, a)\right\}$. Then a standard argument shows $\psi$ is measurable (Hildenbrand, 1974, p. 134). The local nonsatiation property of preferences and the integrability of $f$ imply $\int_{S} \operatorname{proj}_{C} \psi(\mathbf{d}, a) d \nu(a) \neq \emptyset$ by a measurable selection theorem (Hildenbrand, 1974, D.II.2 Theorem 1, p. 54). Let $Z=\cup\left\{\int_{S} \operatorname{proj}_{C} \psi(\mathbf{d}, a) d \nu(a)-Y+Q(\mathbf{d}, S): S \in \mathcal{A} \nu(S)>0\right\}$. We claim $0 \notin Z$. Suppose $0 \in Z$. Then there exist $S \in \mathcal{A}, \nu(S)>0$, and $\tilde{g}(\mathbf{d}, \cdot) \in \psi(d, \cdot)$ such that $\int_{S} \operatorname{proj}_{C} \tilde{g}(\mathbf{d}, a) d \nu(a) \in Y-Q(\mathbf{d}, S)$. Then $(\mathbf{d}, g, S)$ can improve upon $(\mathbf{d}, f)$ since $\tilde{g}(\mathbf{d}, a) \succ_{a}^{\mathbf{d}} \tilde{f}(\mathbf{d}, a)$ a.e. in $S$. Hence, $0 \notin Z$. Note that $Q(\mathbf{d}, S)=\nu(S) \times \mathcal{Q}(\mathbf{d})$. By Vind's lemma (Hildenbrand, 1974, D.II.4 Proposition 5, p. 62), $Z$ is convex. By a separation theorem, there exists $p \in \Re^{I} \times \Re^{2 J}$ s.t. $p \neq 0,0 \leq p \cdot z$ for all $z \in Z$. In fact, $p>0$, for if not there exists $z \in Z$ s.t. $p \cdot z<0$ by the free disposal property in $Q$. Moreover, $p \cdot Z \geq 0$ implies $p \in Y^{*}$. Therefore, without loss of generality, we can normalize $p \in \triangle^{*}$.

Hence, for every $S \in \mathcal{A}, \nu(S)>0$, we have $p \cdot y \leq p \cdot x$ for every $y \in Y-Q(\mathbf{d}, S)$ and $x \in \int_{S} \operatorname{proj}_{C} \psi(\mathbf{d}, a) d \nu(a)$. Then we obtain $p \cdot y \leq p \cdot x$ for every $y \in Y-Q(\mathbf{d}, S)$ and $x \in \int_{S}$ $\operatorname{proj}_{C} \psi(\mathbf{d}, a) d \nu(a)$. Hence, $p \cdot(Y-Q(\mathbf{d}, S)) \leq \Pi(\mathbf{d}, S, p) \leq \inf \left(p \cdot \int_{S} \operatorname{proj}_{C} \psi(\mathbf{d}, a) d \nu(a)\right)$. By interchangeability of infimum and integration (Hildenbrand, 1974, D.II.4 Proposition 6, p. 63), we obtain $\int_{S} \pi(\mathbf{d}, p) d \nu=\Pi(\mathbf{d}, S, p) \leq \int_{S} \inf \left\{p \cdot\left(\operatorname{proj}_{C} \psi(\mathbf{d}, a)\right)\right\} d \nu(a)$. Hence, $\pi(d, p) \leq p \cdot x$ for every $x \in \operatorname{proj}_{C} \psi(\mathbf{d}, a)$ a.e. in $A$.

Now we claim $p \cdot\left(\operatorname{proj}_{C} \tilde{f}(\mathbf{d}, a)\right)=-\min p \cdot Q(\mathbf{d}, A)$ a.e. in $A$. Since $\int_{S} \operatorname{proj}_{C} \psi(\mathbf{d}, a) d \nu(a) \neq$ $\emptyset, \Pi(\mathbf{d}, S, p)<\infty$ and $\pi(\mathbf{d}, p)<\infty$. This implies $\pi(\mathbf{d}, p)=-\min p \cdot Q(\mathbf{d}, A)$, since $Y$ is a convex cone with vertex at the origin. Actually the minimum exists, since the feasible $Q(\mathbf{d}, A)$ are bounded and feasibility is satisfied under $p$. Local nonsatiation implies $p \cdot\left(\operatorname{proj}_{C} \tilde{f}(\mathbf{d}, a)\right) \geq \pi(\mathbf{d}, p)$ a.e. in $A$. Suppose that there exists $S \in \mathcal{A}, \nu(S)>0$, such that $\int_{S} \pi(\mathbf{d}, p) d \nu(a)<p \cdot \int_{S} \operatorname{proj}_{C} \tilde{f}(\mathbf{d}, a) d \nu(a)$. Then there exists $\bar{y} \in Y$ such that $\int_{S} \operatorname{proj}_{C} \tilde{f}(\mathbf{d}, a) d \nu(a) \in\{\bar{y}\}-Q(\mathbf{d}, S)$, and $p \cdot \bar{y}>0$, which contradicts profit maximization by firms. Therefore, $p \cdot\left(\operatorname{proj}_{C} \tilde{f}(\mathbf{d}, a)\right)=\pi(\mathbf{d}, p)$ a.e. in $A$.

So far, we have proved that $\tilde{f}$ attains minimum expenditure a.e. in $A$, i.e., $(\mathbf{d}, f, y, q$, $p$ ) is an expenditure minimizing equilibrium (see Hildenbrand (1968)). However, a minimum expenditure consumption plan is not in general the most preferred plan in the budget set: $B(\mathbf{d}, a, p)=\left\{x \in \tilde{X}(\mathbf{d}, a): p \cdot\left(\operatorname{proj}_{C} x\right) \leq-\pi(\mathbf{d}, p)\right\}$. To prove that an expenditure minimizing equilibrium is an equilibrium with market utilization fees, we need to make use of the boundary condition and local nonsatiation. Consider first the set of $a \in A$ such that $\inf p \cdot\left(\operatorname{proj}_{C} \tilde{X}_{j}(\mathbf{d}, a)\right) \geq \pi(\mathbf{d}, p)$ for all $j \in J$. Then, $p \cdot\left(\operatorname{proj}_{C} \tilde{f}(\mathbf{d}, a)\right)=$ min $p \cdot\left(\operatorname{proj}_{C} \tilde{X}_{j}(\mathbf{d}, a)\right)$, which implies by the boundary condition that $\tilde{f}(\mathbf{d}, a)$ is a maximal element in the budget set as long as $\tilde{f}(\mathbf{d}, a)$ is in the budget set. Next consider the set of $a \in A$ such that there exists $j \in J$ such that inf $p \cdot\left(\operatorname{proj}_{C} \tilde{X}_{j}(\mathbf{d}, a)\right)<\pi(\mathbf{d}, p)$. By the boundary condition, $\operatorname{proj}_{J} \tilde{f}(\mathbf{d}, a) \in\left\{j^{\prime} \in J: \inf p \cdot\left(\operatorname{proj}_{C} \tilde{X}_{j^{\prime}}(\mathbf{d}, a)\right)<\pi(\mathbf{d}, p)\right\}$. We claim that $\tilde{f}(\mathbf{d}, a)$ is a maximal element in the budget set under consumer $a$ 's pref-

distinguish between these two by the context of usage. 
erence order. To show this, let $B_{j}(\mathbf{d}, a, p)=\left\{x \in \tilde{X}_{j}(\mathbf{d}, a): p \cdot\left(\operatorname{proj}_{C} x\right) \leq \pi(\mathbf{d}, a)\right\}$, and let $B_{j}^{\circ}(\mathbf{d}, a, p)=\left\{x \in \tilde{X}_{j}(\mathbf{d}, a): p \cdot\left(\operatorname{proj}_{C} x\right)<\pi(\mathbf{d}, a)\right\}$. Note that $B_{j}(\mathbf{d}, a, p)$ and $B_{j}^{\circ}(\mathbf{d}, a, p)$ denote the budget set of consumer $a$ at location $j$, and the interior of this set, respectively. Obviously, $B(\mathbf{d}, a, p)=\cup_{j \in J} B_{j}(\mathbf{d}, a, p)$ holds. Without loss of generality, let $j=\operatorname{proj}_{J} \tilde{f}(\mathbf{d}, a)$. Suppose that there exist $j^{\prime} \in J$ and $x^{\prime} \in B_{j^{\prime}}(\mathbf{d}, a, p)$ s.t. $x^{\prime} \succ_{a}^{\mathbf{d}} \tilde{f}(\mathbf{d}, a)$. Then $j^{\prime} \in\left\{j^{\prime \prime} \in J: p \cdot\left(\operatorname{proj}_{C} \tilde{X}_{j^{\prime \prime}}(\mathbf{d}, a)\right)>\pi(\mathbf{d}, p)\right\}$ by the boundary condition. Pick a convergent sequence $\left\{x_{k}^{\prime}\right\}_{k=1}^{\infty} \subset B_{j^{\prime}}^{\circ}(\mathbf{d}, a, p)$ s.t. $x_{k}^{\prime} \rightarrow x^{\prime}$. Since $R(\mathbf{d}, a)$ is a closed complete preorder, $P^{a}(\mathbf{d}, a)$ is open. Therefore, there exists an integer $\bar{k}$ s.t. for any $k>\bar{k}$, $x_{k}^{\prime} \succ_{a}^{\mathbf{d}} \tilde{f}(\mathbf{d}, a)$, a contradiction. Hence, $\tilde{f}(\mathbf{d}, a)$ is a maximal element in the budget set under consumer $a$ 's preference order. This proves $\operatorname{Core}(\mathbf{d})=E(\mathbf{d})$.

\subsection{Proof of Corollary 1.}

By the definition of Core and Core $(\mathbf{d})$, Core $\subset \cup_{K}$ Core $(\mathbf{d})$. From Theorem 1, Core $\subset$ $\cup_{K} E(\mathbf{d})$. The proof is completed by applying the definition of $E(\mathbf{d})$.

\subsection{Proof of Theorem 3.2.}

This proof is a combination of the proofs of Hildenbrand (1974, II.2.2 Theorem 2, p. 151) and Hildenbrand (1974, II.4.2 Theorem 2, p. 219), greatly complicated by the use of disconnected consumption sets.

A standard argument proves that the feasible average translated trading set and feasible production set are bounded. Truncate the net production set (the total setup cost is subtracted) $Y-Q(\mathbf{d}, A)$ by a compact hypercube in $C$ that contains the feasible production set in its interior. Denote the net truncated production set by $\hat{Y}(\mathbf{d})$. Next truncate individual translated trading sets in the following way: $\tilde{X}^{k}(\mathbf{d}, a)$ is defined such that $\operatorname{proj}_{C} \tilde{X}_{j}^{k}(\mathbf{d}, a)=$ $\operatorname{proj}_{C} \tilde{X}_{j}(\mathbf{d}, a) \cap(Y+k e)$, for $j \in J$ and $k=1,2, \ldots$, where $e=(1,1, \ldots, 1) \in C$. Since $\mathbf{d} \in K$ is feasible, $\tilde{X}^{k}(\mathbf{d}, a) \neq \emptyset$ a.e. in $A$ for all $k$.

Let $\triangle^{*}=\triangle \cap Y^{*}$ where $Y^{*}$ denotes the dual cone of $Y$. Clearly, $\triangle^{*}$ is compact and convex. Let $\eta(\mathbf{d}, \cdot): \triangle^{*} \rightarrow \hat{Y}(\mathbf{d})$ be such that $\eta(\mathbf{d}, p)=\left\{y \in \hat{Y}(\mathbf{d}): p \cdot y \geq p \cdot y^{\prime}\right.$ for any $\left.y^{\prime} \in \hat{Y}(\mathbf{d})\right\}$. Then, $\eta(\mathbf{d}, \cdot)$ has a closed graph, and is nonempty-, compact- and convexvalued. Also let $\pi(\mathbf{d}, \cdot): \triangle^{*} \rightarrow \Re$ be such that $\pi(\mathbf{d}, p)=p \cdot \eta(\mathbf{d}, p)$. Then, $\pi(\mathbf{d}, \cdot)$ is a continuous function.

Let us move to the consumer sector. Let $B^{k}(\mathbf{d}, a, p)=\left\{x \in \operatorname{proj}_{C} \tilde{X}^{k}(\mathbf{d}, a): p \cdot x \leq\right.$ $\pi(\mathbf{d}, p)\}, B^{k}(\mathbf{d}, a, p)=\cup_{J} B_{j}^{k}(\mathbf{d}, a, p)$, and $B_{V}^{k}(\mathbf{d}, a, p)=\cup_{V} B_{j}^{k}(\mathbf{d}, a, p)$ for $V \subset J$ such that $V \neq \emptyset$. These denote budget relations in some (or all) locations. Let $\varphi^{k}: K \times A \times \triangle^{*} \rightarrow$ $\tilde{X}^{k}(\mathbf{d}, a)$ be such that $\varphi^{k}(\mathbf{d}, a, p)=\left\{x \in B^{k}(\mathbf{d}, a, p): x \succeq_{a}^{\mathbf{d}} x^{\prime}\right.$ for any $\left.x^{\prime} \in B^{k}(\mathbf{d}, a, p)\right\}$. $\varphi$ denotes consumer $a$ 's demand relation when the consumption set is truncated by $Y+$ ke. Since $(Y-Q(\mathbf{d}, A)) \cap \operatorname{proj}_{C} \tilde{X}(\mathbf{d}, a) \neq \emptyset$ a.e. in $A$, there exists $j \in J$ such that $\hat{Y}(\mathbf{d}) \cap \operatorname{proj}_{C} \tilde{X}_{j}^{k}(\mathbf{d}, a) \neq \emptyset$ for all $k$ a.e. in $A$. Since $p \in \triangle^{*}$, there exists $j \in J$ such that $B_{j}^{k}(\mathbf{d}, a, p) \neq \emptyset$ a.e. in $A$. 
Let $\triangle^{\circ}=\left\{p \in \triangle^{*}: p^{c} \gg 0\right\}$, where $p^{c}$ is commodity price vector. Eventually, we will show that the equilibrium price relative to $k$ is in $\triangle^{\circ}$. Given $a \in A, \mathbf{d} \in K, p \in \triangle^{\circ}$, we partition $J$ into three sets:

$$
\begin{aligned}
& J_{>}(\mathbf{d}, a, p)=\left\{j \in J: \pi(\mathbf{d}, p)>\min p \cdot\left[\operatorname{proj}_{C} \tilde{X}_{j}^{k}(\mathbf{d}, a)\right]\right\}, \\
& J_{=}(\mathbf{d}, a, p)=\left\{j \in J: \pi(\mathbf{d}, p)=\min p \cdot\left[\operatorname{proj}_{C} \tilde{X}_{j}^{k}(\mathbf{d}, a)\right]\right\}, \\
& J_{<}(\mathbf{d}, a, p)=\left\{j \in J: \pi(\mathbf{d}, p)<\min p \cdot\left[\operatorname{proj}_{C} \tilde{X}_{j}^{k}(\mathbf{d}, a)\right]\right\} .
\end{aligned}
$$

Since $\mathbf{d}$ is feasible, $J_{>}(\mathbf{d}, a, p) \neq \emptyset$ a.e. in $A$. Given $a \in A, \mathbf{d} \in K$, and $j \in J$, we partition $\triangle^{\circ}$ into three sets in a similar manner:

$$
\begin{aligned}
& \triangle_{>}^{\circ}(\mathbf{d}, a, j)=\left\{p \in \triangle^{\circ}: \pi(\mathbf{d}, p)>\min p \cdot\left[\operatorname{proj}_{C} \tilde{X}_{j}^{k}(\mathbf{d}, a)\right]\right\}, \\
& \triangle_{=}^{\circ}(\mathbf{d}, a, j)=\left\{p \in \triangle^{\circ}: \pi(\mathbf{d}, p)=\min p \cdot\left[\operatorname{proj}_{C} \tilde{X}_{j}^{k}(\mathbf{d}, a)\right]\right\}, \\
& \triangle_{<}^{\circ}(\mathbf{d}, a, j)=\left\{p \in \triangle^{\circ}: \pi(\mathbf{d}, p)<\min p \cdot\left[\operatorname{proj}_{C} \tilde{X}_{j}^{k}(\mathbf{d}, a)\right]\right\} .
\end{aligned}
$$

Obviously $\triangle_{>}^{\circ}(\mathbf{d}, a, j)$ is open relative to $\triangle^{\circ}$ It is easy to show that $B_{j}(\mathbf{d}, a, \cdot)$ is continuous on $\triangle_{>}^{\circ}(\mathbf{d}, a, j)$.

Now, we claim $\varphi^{k}(\mathbf{d}, a, \cdot)$ has a closed graph in $\triangle^{\circ}$. Since $B^{k}(\mathbf{d}, a, \cdot)$ is not continuous in general due to disconnected consumption sets, we need several steps to prove this. First note that if $j \in J_{>}(\mathbf{d}, a, p)$, there exists $x \in B_{j}^{k}(\mathbf{d}, a, p)$ such that $x \succeq_{a}^{\mathbf{d}} x^{\prime}$ for $x^{\prime} \in B_{j^{\prime}}^{k}(\mathbf{d}, a, p)$ with $j^{\prime} \in J_{=}(\mathbf{d}, a, p)$ by the boundary condition. Hence if $J_{>}(\mathbf{d}, a, p) \neq \emptyset$, then $\operatorname{proj}_{J} \varphi^{k}(\mathbf{d}, a, p) \in$ $J_{>}(\mathbf{d}, a, p)$.

Second we show that if $J_{>}(\mathbf{d}, a, p) \neq \emptyset$, then for any $p \in \triangle_{=}^{\circ}(\mathbf{d}, a, j)$, for any sequence $\left\{p^{s}\right\}_{s=1}^{\infty} \rightarrow p$ such that $\left\{p^{s}\right\}_{s=1}^{\infty} \subset \triangle_{>}^{o}(\mathbf{d}, a, j)$, there exists an integer $\bar{s}$ such that for any $s \geq \bar{s}, j \notin \operatorname{proj}_{J} \varphi^{k}\left(\mathbf{d}, a, p^{s}\right)$. To show this, let $x \in B_{j}^{k}(\mathbf{d}, a, p)$ and $x^{\prime} \in B_{j^{\prime}}^{k}(\mathbf{d}, a, p)$ where $j^{\prime} \in J_{>}(\mathbf{d}, a, p)$. From local nonsatiation and the boundary condition, $x^{\prime} \succ_{a}^{\mathbf{d}} x$. Since $P(\mathbf{d}, a)$ is open, the statement is proved.

Third, let $\triangle_{>}^{\circ}(\mathbf{d}, a, V)=\left(\cap_{V} \triangle_{>}^{\circ}(\mathbf{d}, a, j)\right) \backslash\left(\cup_{J \backslash V} \triangle_{>}^{\circ}(\mathbf{d}, a, j)\right)$ for $V \subset J$ with $V \neq \emptyset$, where $\backslash$ is set subtraction. Then, $\triangle^{\circ}$ is partitioned by $\triangle_{>}^{\circ}(\mathbf{d}, a, V)$ for $V \subset J$ such that $V \neq \emptyset$. Let $\varphi_{V}^{k}(\mathbf{d}, a, p)=\left\{x \in B_{V}^{k}(\mathbf{d}, a, p): x \succeq_{a}^{\mathbf{d}} x^{\prime}\right.$ for any $\left.x^{\prime} \in B_{V}^{k}(\mathbf{d}, a, p)\right\}$. Since $B_{V}^{k}(\mathbf{d}, a, \cdot)$ is continuous in $\triangle_{>}^{\circ}(\mathbf{d}, a, V), \varphi_{V}^{k}(\mathbf{d}, a, \cdot)$ has a closed graph in $\triangle_{>}^{\circ}(\mathbf{d}, a, V)$ for any $V \subset J$ (Hildenbrand, 1974, II.1.2 Corollary 2, p. 104). Since $R(\mathbf{d}, a)$ is a complete preorder, $\varphi^{k}(\mathbf{d}, a, p)=\varphi_{V}^{k}(\mathbf{d}, a, p)$ for any $p \in \triangle_{>}^{\circ}(\mathbf{d}, a, V)$, for any $V \subset J$ (see Konishi, 1996, Lemma 2). Hence, $\varphi^{k}(\mathbf{d}, a, \cdot)$ has a closed graph in $\triangle_{>}^{\circ}(\mathbf{d}, a, V)$ for any $V \subset J$.

Fourth, let $\left\{p^{s}\right\}_{s=1}^{\infty} \subset \triangle_{>}^{\circ}(\mathbf{d}, a, V)$ be such that $j \in V$ and $p^{s} \rightarrow p \in \triangle^{\circ} \backslash \triangle_{>}^{\circ}(\mathbf{d}, a, j)$. Then, if $x^{s} \rightarrow x$ such that $x^{s} \in \varphi^{k}\left(\mathbf{d}, a, p^{s}\right)$ for each $s=1,2, \ldots$, then for $s$ large enough $j \notin \operatorname{proj}_{J} x^{s}$ by the argument in the second step above.

These four steps above prove that $\varphi^{k}(\mathbf{d}, a, \cdot)$ has a closed graph on $\triangle^{\circ}$.

Now, let $\Psi^{k}(\mathbf{d}, p)=\int_{A} \operatorname{proj}_{C} \varphi^{k}(\mathbf{d}, a, p) d \nu(a)$. Since $\varphi^{k}(\mathbf{d}, \cdot, p)$ is measurable and integrably bounded, $\Psi^{k}(\mathbf{d}, p) \neq \emptyset$ (Hildenbrand, 1974, D.II.4 Theorem 2, p. 62). Furthermore, 
$\Psi^{k}(\mathbf{d}, \cdot)$ has a closed graph on $\triangle^{\circ}$ (Hildenbrand, 1974, D.II.4 Proposition 8, p. 73), and is convex-valued (Hildenbrand, 1974, D.II.4 Theorem 3, p. 62).

Let $Z^{k}=\int_{A} \operatorname{proj}_{C} X^{k}(\mathbf{d}, a) d \nu(a)-\hat{Y}(\mathbf{d})$. Then, $Z^{k}$ is compact and convex. Let $\triangle^{s}=$ $\left\{p \in \triangle^{*}: p_{i}^{c} \geq 1 / s\right.$ for all $\left.i \in I\right\}$. Clearly, $\triangle^{s}$ is compact and convex. For $s$ large enough, $\triangle^{s} \neq \emptyset$, and $\triangle^{s} \subset \triangle^{\circ}$. Let $\zeta^{k s}: \triangle^{s} \rightarrow Z^{k}$ be such that $\zeta^{k s}(p)=\Psi^{k}(\mathbf{d}, p)-\eta(\mathbf{d}, p)$. Then $\zeta^{k s}$ has a closed graph, and is convex-valued. Let $\theta^{k s}: Z^{k} \rightarrow \triangle^{s}$ be such that $\theta^{k s}(z)=\left\{p \in \triangle^{s}: p \cdot z \geq p^{\prime} \cdot z\right.$ for any $\left.p^{\prime} \in \triangle^{s}\right\}$. Then, $\theta^{k s}$ has a closed graph, and is convex-valued. Let $\xi^{k s}=\zeta^{k} \times \theta^{k s}: \triangle^{s} \times Z^{k} \rightarrow \triangle^{s} \times Z^{k}$. Then $\xi^{k s}$ has a fixed point by Kakutani's theorem. Hence, there exists a list $\left(\mathbf{d}, f^{k s}, y^{k s}, p^{k s}\right)$ such that $\left(\mathbf{d}, f^{k s}\right) \in \mathcal{F}$, $y^{k s} \in \hat{Y}(\mathbf{d})$, and $p^{k s} \in \triangle^{s}$ satisfying the following conditions: (i) $\triangle^{k s}(\mathbf{d}, a) \in \varphi^{k}\left(\mathbf{d}, a, p^{k s}\right)$ a.e. in $A$, (ii) $y^{k s} \in \eta\left(\mathbf{d}, p^{k s}\right)$, and (iii) $\int_{A} \operatorname{proj}_{C} \tilde{f}^{k s}(\mathbf{d}, a) d \nu(a)-y^{k s} \leq 0$.

Pick a sequence of fixed points $\left\{\left(\mathbf{d}, f^{k s}, y^{k s}, p^{k s}\right)\right\}_{k=1}^{\infty}$. From (i) and (iii), we obtain $\int_{A} \operatorname{proj}_{C} \tilde{f}^{k s}(\mathbf{d}, a) d \nu(a) \in \hat{Y}(\mathbf{d})$ for each $k$. Then, without loss of generality (due to compactness), we can assume $\int_{A} \operatorname{proj}_{C} \tilde{f}^{k s}(\mathbf{d}, a) d \nu(a) \rightarrow x^{s}, y^{k s} \rightarrow \bar{y}^{s}$, and $p^{k s} \rightarrow p^{s}$. Since $\eta(\mathbf{d}, \cdot)$ is closed, $\bar{y}^{s} \in \eta\left(\mathbf{d}, p^{s}\right)$. From Fatou's lemma in several dimensions (Hildenbrand, 1974, D.II.4 Lemma 3, p. 69), there exists $f^{s}$ such that $\left(\mathbf{d}, f^{s}\right) \in \mathcal{F}$ such that (i) $\tilde{f}^{s}(\mathbf{d}, a)$ is an accumulation point of $\left\{\tilde{f}^{k s}(\mathbf{d}, a)\right\}_{k=1}^{\infty}$ a.e. in $A$, (ii) $\int_{A} \operatorname{proj}_{C} \tilde{f}^{s}(\mathbf{d}, a) d \nu(a) \leq x^{s}$.

Since $\tilde{f}^{k s}(\mathbf{d}, a) \in \varphi^{k}\left(p^{k s}\right)$ a.e. in $A$, and since $P(\mathbf{d}, a)$ is open, $\tilde{f}^{s}(\mathbf{d}, a) \in \varphi\left(\mathbf{d}, a, p^{s}\right)$ a.e. in $A$, where $\varphi(\mathbf{d}, a, p)=\left\{x \in B(\mathbf{d}, a, p): x \succeq_{a}^{\mathbf{d}} x^{\prime}\right.$ for any $\left.x^{\prime} \in B(\mathbf{d}, a, p)\right\}$. Let $y^{s}=$ $\int_{A} \operatorname{proj}_{C} \tilde{f}^{s}(\mathbf{d}, a) \nu(a)$. We will prove that $y^{s} \in \eta\left(\mathbf{d}, p^{s}\right)$. To accomplish this, let $z^{s}=y^{s}-\bar{y}^{s}$. Since local nonsatiation of preferences is implied by our assumptions, $p^{s} \cdot\left(\operatorname{proj}_{C} \tilde{f}^{s}(\mathbf{d}, a)\right)=$ $\pi\left(\mathbf{d}, p^{s}\right)$ a.e. in $A$. Hence $p^{s} \cdot y^{s}=p^{s} \cdot \bar{y}^{s}$. We show $y^{s} \in Y-Q(\mathbf{d}, A)$. It suffices to show $z^{s} \leq 0$. Since $z^{s}=\left(\lim _{k \rightarrow \infty} \int_{A} \operatorname{proj}_{C} \tilde{f}^{k s}(\mathbf{d}, a) d \nu(a)-\bar{y}^{s}\right)-\left(\lim _{k \rightarrow \infty} \int_{A} \operatorname{proj}_{C} \tilde{f}^{k s}(\mathbf{d}, a) d \nu(a)-\right.$ $\left.\int_{A} \operatorname{proj}_{C} \tilde{f}^{s}(\mathbf{d}, a) d \nu(a)\right), z^{s} \leq 0$ follows by Fatou's lemma in several dimensions and the free disposal property of $Y-Q(\mathbf{d}, A)$. Hence $\left(\mathbf{d}, f^{s}, y^{s}, p^{s}\right)$ is an equilibrium with participation fees relative to $s$.

Now, what is left to show is that for $s$ large enough, $p_{i}^{s}>1 / s$ for all $i \in I$. If there exists such an $s,\left(\mathbf{d}, f^{s}, y^{s}, p^{s}\right) \in E(\mathbf{d})$ holds. Suppose not. From interiority and monotonicity in commodity consumption, we can find $s$ such that $z_{i}^{s}>0$ for some $i \in I$ (Hildenbrand, 1974, II.2.2 Lemma 1, p. 150, and II.1.3 Proposition 6, p. 119). This contradicts $z^{s} \leq 0$ for all $s$.

\subsection{Proof of Corollary 2.}

From the boundary condition and the feasibility of $\mathbf{d}$, a.e. in $A$ consumer a has no incentive to deviate from the grand coalition, which proposes $(\mathbf{d}, f) \in E(\mathbf{d})$. Hence $(\mathbf{d}, f) \in$ Core $(\mathbf{d})$ by Theorem 1. Theorem 2 proves $E(\mathbf{d}) \neq \emptyset$ if interiority is satisfied. If interiority is not satisfied, then by the boundary condition, a.e. in $A$ consumer $a$ can only consume a least preferred consumption plan. Hence, there is no incentive to deviate from such an allocation, and $\operatorname{Core}(\mathbf{d}) \neq \emptyset$. 


\subsection{Proof of Theorem 4.1.}

First note that $Y \cap \operatorname{proj}_{C} X(a) \neq \emptyset$, i.e., there exists a feasible allocation $(\emptyset \in K)$. Note also that by the assumption that the set of feasible marketplace structures is bounded, the set of feasible marketplace structures $\operatorname{proj}_{K} \mathcal{F}$ is compact. To show this, it is enough to recall that the function $\delta^{j}$ is a continuous function on $(K, \mathcal{O})$, and recall the assumptions on $Q$, $Y$, and $X$. Denote the feasible marketplace structures by $\hat{K}$.

Suppose that $\hat{K}=\{\emptyset\}$. Then, obviously $(\emptyset, f) \in$ Core, where everybody is getting one of the least preferable consumption plans by the boundary condition (see footnote 17). Thus, we can concentrate on the case where $\hat{K} \neq\{\emptyset\}$. In this case, there is $\mathbf{d} \in \hat{K}$ where there is a feasible allocation: i.e., $(Y-Q(\mathbf{d}, A)) \cap \operatorname{proj}_{C} \tilde{X}(\mathbf{d}, a) \neq \emptyset$ a.e. in $A$ so that the assumptions of Corollary 2 are satisfied.

Since we assume that consumers are identical almost everywhere, we drop a from the notation for preference relations and consumption sets. Since $\succeq$ is a continuous complete preorder, there exists a continuous utility representation of $\succeq, u: X \rightarrow[0,1]$. By the definition of $F$, we can naturally define $\tilde{u}: F \rightarrow[0,1]$ such that if $u(f(a))=u^{*}$ a.e. in $A$, then $\tilde{u}(f)=u^{*}$. Clearly, there exists $\sup \left\{\tilde{u}(f): f \in \operatorname{proj}_{X} F \cap \operatorname{proj}_{X} \mathcal{F}\right\}=\bar{u}$. Then, there exists a sequence $\left\{\left(\mathbf{d}^{s}, f^{s}\right)\right\}_{s=1}^{\infty}$ such that $\tilde{u}\left(f^{s}\right) \rightarrow \bar{u}$. Since the feasible average consumption set is bounded and $\hat{K}$ is compact, there exists a convergent subsequence of $\left\{\left(\mathbf{d}^{s}, \int_{A} \operatorname{proj}_{C} f^{s}(a) d \nu(a)\right)\right\}_{s=1}^{\infty} \rightarrow(\mathbf{d}, \bar{x})$. Since $\hat{K}$ is compact, $\mathbf{d} \in \hat{K}$. Since $Y-Q(\mathbf{d}, A)$ is closed, $\bar{x} \in Y-Q(\mathbf{d}, A)$. From Fatou's lemma in several dimensions, there exists $(\mathbf{d}, f) \in \mathcal{F}$, such that (i) $\int_{A} \operatorname{proj}_{C} f d \nu \leq \bar{x}$, and (ii) $f(a)$ is an accumulation point of $\left\{f^{s}(a)\right\}_{s=1}^{\infty}$ a.e. in $A$. Since $\mathbf{d}^{s} \rightarrow \mathbf{d}, \# \mathbf{d}^{s}=\# \mathbf{d}$. Since $\delta_{j}$ is continuous and $X$ is closed, $f(a) \in \tilde{X}(\mathbf{d}, a)$, which is a truncated trading set. From closedness of $\succeq,(\mathbf{d}, f) \in F$ so $(\mathbf{d}, f) \in F \cap \mathcal{F}$. From the continuity of $u, \tilde{u}(f)=\bar{u}$. Hence $(\mathbf{d}, f)$ is a maximal element of $F \cap \mathcal{F}$ in $\succeq_{A}$.

Now we will prove that $(\mathbf{d}, f) \in$ Core. Suppose not. Then, there exist $\left(\mathbf{d}^{\prime}, g\right) \in \mathcal{F}$ and $S \in \mathcal{A}, \nu(S)>0$, such that $g(a) \succ f(a)$ a.e. in $S$. Since $\mathbf{d}^{\prime} \in \operatorname{proj}_{K} \mathcal{F}, \operatorname{Core}\left(\mathbf{d}^{\prime}\right) \neq \emptyset$ by Corollary 2. Let $\left(\mathbf{d}^{\prime}, h\right) \in$ Core $\left(\mathbf{d}^{\prime}\right)$. Then, $(g, S)$ cannot improve upon $h$. This implies that there exists $S^{\prime} \in \mathcal{A}, S^{\prime} \subset S, \nu\left(S^{\prime}\right)>0$, such that $h(a) \succeq g(a)$ a.e. in $S^{\prime}$. Since $h \in F$ by Corollary $1, h(a) \succ f(a)$ a.e. in $A$ by transitivity of $\succeq$. This contradicts $\tilde{u}(f)=\bar{u}$. Hence $(\mathbf{d}, f) \in$ Core.

\subsection{Proof of Corollary 3}

Since Core $\in P$, Core $\subset F \cap P$ (Corollary 1$)$. Let $(\mathbf{d}, f) \in F \cap P$. Then, $\tilde{u}(f)=\max \left\{\tilde{u}\left(f^{\prime}\right)\right.$ : $\left.\left(\mathbf{d}^{\prime}, f^{\prime}\right) \in F \cap \mathcal{F}\right\}$, which implies $(\mathbf{d}, f) \in$ Core by the argument in the proof of Theorem 4.1.

\section{References}

[1] Baesemann, R., 1977, The formation of small market places in a competitive economic process - the dynamics of agglomeration, Econometrica 45, 361-374. 
[2] Baruchov, E. and O. Hochman, 1977, Optimum and market equilibrium in a model of a city without a predetermined center, Environment and Planning A 9, 849-856.

[3] Beckmann, M. and J.-F. Thisse, 1986, The location of production activities, in: $P$. Nijkamp, ed., Handbook of Regional and Urban Economics: Volume 1, Regional Economics (North-Holland, Amsterdam) 21-95.

[4] Berliant, M., 1985, Equilibrium models with land: A criticism and an alternative, Regional Science and Urban Economics 15, 325-340.

[5] Berliant, M. and M. Fujita, 1992, Alonso's discrete population model of land use: Efficient allocations and competitive equilibria, International Economic Review 33, 535-566.

[6] Berliant, M. and T. ten Raa, 1991, On the continuum approach of spatial and some local public goods or product differentiation models: Some problems, Journal of Economic Theory 55, 95-120.

[7] Berliant, M. and T. ten Raa, 1994, Regional science: The state of the art, Regional Science and Urban Economics 24, 631-647.

[8] Berliant, M. and P. Wang, 1993, Endogenous formation of a city without agglomerative externalities or market imperfections: Marketplaces in a regional economy, Regional Science and Urban Economics 23, 121-144.

[9] Ciccone A. and R.E. Hall, 1997, Productivity and the density of economic activity, American Economic Review 86, 54-70.

[10] Cronon, W., 1991, Nature's Metropolis: Chicago and the Great West (W.W. Norton and Company, New York).

[11] Diamantaras, D., and R.P. Gilles, 1996, The pure theory of public goods: Efficiency, decentralization and the core, International Economic Review 37, 851-860.

[12] Diamantaras, D., R.P. Gilles, and S. Scotchmer, 1996, Decentralization of Pareto optima in economies with public projects and nonessential private goods, Economic Theory 8 , $555-564$.

[13] Dixit, A. and J. Stiglitz, 1977, Monopolistic competition and optimum product diversity, American Economic Review 67, 297-308.

[14] Ellickson, B. and W. Zame, 1991, The market for land, mimeo (incomplete draft).

[15] Ellickson, B. and W. Zame, 1994, Foundations for a competitive theory of economic geography, mimeo (incomplete draft).

[16] Foley, D., 1970, Lindahl's solution and the core of an economy with public goods, Econometrica 38, 66-72. 
[17] Fujita, M., 1986, Urban land use theory, in: R. Arnott, ed., Location Theory (Harwood Academic Publishers, Chur) 73-150.

[18] Fujita, M., 1988, A monopolistic competition model of spatial agglomeration: Differentiated product approach, Regional Science and Urban Economics 18, 87-124.

[19] Fujita, M. and P. Krugman, 1995, When is the economy monocentric? Von Thünen and Chamberlin unified, Regional Science and Urban Economics 25, 505-528.

[20] Fujita, M. and H. Ogawa, 1982, Multiple equilibria and structural transition of nonmonocentric urban configurations, Regional Science and Urban Economics 12, 161-196.

[21] Hartwick, P. and J. Hartwick, 1974, Efficient resource allocation in a multinucleated city with intermediate goods, Quarterly Journal of Economics 88, 340-352.

[22] Hendricks, K., M. Piccione, and G. Tan, 1995, The economics of hubs: The case of monopoly, The Review of Economic Studies 62, 83-99.

[23] Hildenbrand, W., 1968, The core of an economy with a measure space of economic agents, The Review of Economic Studies 35, 443-452.

[24] Hildenbrand, W., 1974, Core and Equilibria of a Large Economy (Princeton University Press, Princeton, NJ).

[25] Imai, H., 1982, CBD hypothesis and economies of agglomeration, Journal of Economic Theory 28, 275-299.

[26] Jackson, M.O., and A. Wolinsky, 1996, A strategic model of social and economic networks, Journal of Economic Theory 71, 44-74.

[27] Jacobs, J., 1969, The Economy of Cities (Random House, New York).

[28] Jacobs, J., 1984, Cities and the Wealth of Nations (Random House, New York).

[29] Kim, S., 1995, Expansion of markets and the geographic distribution of economic activities: The trends in U.S. regional manufacturing structure, 1860-1987, Quarterly Journal of Economics 110, 881-908.

[30] Konishi, H., 1996, Voting with ballots and feet: Existence of equilibrium in a local public good economy, Journal of Economic Theory 68, 480-509.

[31] Krugman, P., 1991a, Increasing returns and economic geography, Journal of Political Economy 99, 483-499.

[32] Krugman, P., 1991b, Geography and Trade (MIT Press, Cambridge, MA).

[33] Krugman, P., 1993a, On the number and location of cities, European Economic Review 37, 293-298. 
[34] Krugman, P., 1993b, First nature, second nature, and metropolitan location, Journal of Regional Science 33, 129-144.

[35] Mas-Colell, A., 1977, Indivisible commodities and general equilibrium theory, Journal of Economic Theory 16, 443-456.

[36] Mas-Colell, A., 1980, Efficiency and decentralization in the pure theory of public goods, Quarterly Journal of Economics 94, 625-641.

[37] McKenzie, L., 1959, On the existence of general equilibrium for a competitive market, Econometrica 27, 54-71.

[38] Mills, E., 1967, An aggregative model of resource allocation in a metropolitan area, American Economic Review, Papers and Proceedings 57, 197-210.

[39] Mills, E., 1972, Markets and efficient resource allocation in urban areas, Swedish Journal of Economics 74, 100-113.

[40] Palivos, T. and P. Wang, 1996, Spatial agglomeration and endogenous growth, Regional Science and Urban Economics 26, 645-669.

[41] Papageorgiou, Y., 1979, Agglomeration, Regional Science and Urban Economics 9, 4159.

[42] Papageorgiou, Y. and D. Pines, 1990, The logical foundations of urban economics are consistent, Journal of Economic Theory 50, 37-53.

[43] Papageorgiou, Y.Y. and T.R. Smith, 1983, Agglomeration as local instability of spatially uniform steady-states, Econometrica 51, 1109-1120.

[44] Roberts, D. J., 1974, The Lindahl solution for economies with public goods, Journal of Public Economics 3, 23-42.

[45] Schweizer, U., P. Varaiya and J. Hartwick, 1976, General equilibrium and location theory, Journal of Urban Economics 3, 285-303.

[46] Starrett, D., 1978, Market allocation of location choice in a model with free mobility, Journal of Economic Theory 17, 21-37.

[47] Stuart, C., 1970, Search and the spatial organization of trading, in: S. Lippman and J. McCall, eds., Studies in the Economics of Search (North-Holland, Amsterdam).

[48] Vasil'ev, V., S. Weber, and H. Wiesmeth, 1995, Core equivalence with congested public goods, Economic Theory 6, 373-387.

[49] Wooders, M., 1980, The Tiebout hypothesis: Near optimality in local public good economies, Econometrica 48, 1467-1486. 
[50] Yamazaki, A., 1978, An equilibrium existence theorem without convexity assumptions, Econometrica 46, 541-555. 